\title{
A Fast SVD-Hidden-Nodes Based Extreme Learning Machine for Large-Scale Data Analytic
}

\author{
Wan-Yu Deng ${ }^{\mathrm{a}, \mathrm{b}}$, Zuo Bai ${ }^{\mathrm{c}}$, Guang-Bin Huang ${ }^{\mathrm{c}}$, Qing-Hua Zheng ${ }^{\mathrm{d}}$ \\ ${ }^{a}$ School of Computer, Xian University of Posts \& Telecommunications, Shaanxi, China \\ ${ }^{b}$ School of Computer Engineering, Nanyang Technological University, Singapore \\ ${ }^{c}$ School of Electrical \& Electronic Engineering, Nanyang Technological University, Singapore \\ ${ }^{d}$ Department of Computer Science and Technology, Xi'an Jiaotong University, China
}

\begin{abstract}
Big dimensional data is a growing trend that is emerging in many real world contexts, extending from web mining, gene expression analysis, protein-protein interaction to high-frequency financial data. Nowadays, there is a growing consensus that the increasing dimensionality poses impeding effects on the performances of classifiers, which is termed as the "peaking phenomenon" in the field of machine intelligence. To address the issue, dimensionality reduction is commonly employed as a preprocessing step on the Big dimensional data before building the classifiers. In this paper, we propose an Extreme Learning Machine (ELM) approach for large-scale data analytic. In contrast to existing approaches, we embed hidden nodes that are designed using singular value decomposition (SVD) into the classical ELM. These SVD nodes in the hidden layer are shown to capture the underlying characteristics of the Big dimensional data well, exhibiting excellent generalization performances. The drawback of using SVD on the entire data set, however, is the high computational complexity involved. To address this, a fast divide and conquer approximation scheme is introduced to maintain computational tractability on high volume data. The resultant algorithm proposed is labelled here as Fast Singular Value Decomposition-Hidden-nodes based Extreme Learning Machine or FSVD-H-ELM in short. In FSVD-H-ELM, instead of identifying the SVD hidden nodes directly from the entire data set, SVD hidden nodes are derived
\end{abstract}

\footnotetext{
* Corresponding author

Email address: wanyu. deng@gmail . com (Wan-Yu Deng)
}

Preprint submitted to Neural Networks

August 3, 2015

(C) 2015. This manuscript version is made available under the Elsevier user license http://www.elsevier.com/open-access/userlicense/1.0/ 
from multiple random subsets of data sampled from the original dataset. Comprehensive experiments and comparisons are conducted to assess the FSVD-H-ELM against other state-of-the-art algorithms. The results obtained demonstrated the superior generalization performance and efficiency of the FSVD-H-ELM.

Keywords: extreme learning machine, singular value decomposition, Big data, Big dimensional data, fast approximation method

\section{Introduction}

The notion of "Big Dimensionality" was established recently in [1] to place emphasis on the growing phenomenon of problem dimensionality that is observed across diverse scenarios and applications in the real world. When addressing Big Data, vol5 ume often comes to mind naturally and take immediate precedence, since it presents the challenges pertaining to the scalability issue. Also, this is what directly comes to our mind when we refer to the term "Big". However, it is worth highlighting that researchers have largely considered the "Big Instance Size" factor of "Volume" in the Big Data analytics community, which refers to the massive amounts of data that we continue to produce daily. In the context of data analytics, volume can be defined as a product of instance size and dimensionality of the data. The issue of "Big Dimensionality", however, has received much lesser attention. For more coverage on this relatively under-explored topic of "Big Dimensionality" wherein the explosion of features (variables) brings about new challenges to computational intelligence and Big data, the reader is referred to [1]. Big dimensional data is a growing trend that is emerging in many real world contexts, extending from web mining, gene expression analysis, protein-protein interaction to high-frequency financial dataToday, there is a growing consensus that the increasing dimensionality poses impeding effects on the performances of classifiers, which is termed as the "peaking phenomenon" in the field of machine intelligence [2].Previous methods [3] to address the high dimensionality characteristics of data have mainly concentrated on preprocessing the data with dimensionality reduction methods, keeping only the salient features and discarding the non-informative onesbefore building the classifier. In this paper, we present an attempt 
to address both Big dimensionality and Big instance size simultaneously in the context of Big Data analytic by embedding Singular Value Decomposition Hidden nodes into the classical Extreme Learning Machine.

In spite of the extensive works on ELM research, existing efforts still cannot arrive at representations and models that are elegant for Big data. Like all methods known to date, ELM suffers from the effects of 'curse of dimensionality and instance size'. The performance of ELM has been shown to deteriorate substantially and becomes unstable in the face of Big data. Taking this cue, this paper considers an implicit incorporation of dimension reduction mechanism into the classical ELM to achieve high generalization performances. In particular, we present a Fast Singular Value Decomposition-Hiddennodes based Extreme Learning Machine (or FSVD-H-ELM in short) to cope with Big dimensional \& Big instance data. We embed SVD hidden nodes into the classical Extreme Learning Machine to arrive at SVD-H-ELM and provide theoretical proofs on the optimality of using SVD hidden nodes over random hidden nodes. Further, instead of identifying the SVD hidden nodes directly from the Big dataset, a fast divide and conquer approximation scheme is also introduced to maintain computational tractability and scalability by deriving SVD hidden nodes from multiple random subsets of data sampled from the original Big dataset, in order to arrive at the Fast SVD-H-ELM or FSVD-H-ELM. Comprehensive experiments conducted on commonly used Big dataset indicate that the SVD nodes in the hidden layer are able to capture the underlying characteristics of the Big dataset well, leading to excellent generalization performances and 45 efficiencies.

The rest of the paper is organized as follows: Section II gives a brief overview of the classical ELM. Section III details the proposed Fast Singular Value DecompositionHidden-nodes based Extreme Learning Machine (or FSVD-H-ELM). In Section IV, A discussion on the feature learning ability of the fast divide-and-conquer scheme is made. In Section V, we summarize the experimental studies on twelve commonly used benchmark problems. Section VI summarizes the main conclusions. 


\section{Review of Extreme Learning Machine and Large-scale Data Analytics}

\subsection{Extreme Learning Machine}

Feedforward neural networks play key roles in data analytic and have been widely 55 applied in many applications for its promising generalization ability [4, 5, 6, 7, 8, 9]. However, popular learning techniques face some challenging issues such as intensive human intervene and slow learning speed[10]. Although many effective algorithms, such as the back-propagation are available, training a neural network with all parameters adjustable is usually of high computational burden[11, 12]. To overcome such issue, a useful learning scheme, the extreme learning machine (ELM), was suggested in [13] for single layer feedforward neural networks and subsequently extended to different variations such as local connected structure[14] and multi hidden layer$\mathrm{s}$ structure[15]. ELM and its variations[16, 17, 18, 19, 20, 21, 22, 23] have been successfully used in the fields as object recognition[14],terrain-based navigation [24] activity recognition[25], time series[26], security assessment[27], written character recognition [28], face recognition[29], gene selection and cancer classification [30]. In essence ELM is a learning scheme whose hidden nodes need not be tuned and can be randomly generated. Then the ELM transforms the training of the neural network into a linear problem where the output weights of ELMs can be analytically determined instead of being tuned[31]. For the sake of efficiency, in real applications they may be determined in different ways such as with or without iterations, with or without incremental implementations, etc. Xu etc.[32, 33] studied the theoretical feasibility of ELM and proved that via suitable activation functions, such as polynomials, NadarayaWatson and sigmoid functions, the ELMs can attain the theoretical generalization bound of the neural networks with all connections adjusted. Before ELM, the similar idea has been adopted earlier in [34][35] as the echo state network method (ESN), in [36] as the liquid state machine (LSM), in[37] as RBF network with random centers, in [38] as the feedforward neural networks with random weights and in [39] as the random vector functional-link network (RVFL). The relationships between ELMs and these earlier 80 work please refer to the literatures [40, 41]. 
The output function of ELM with $L$ hidden nodes for generalized SLFNs is:

$$
f_{L}(\mathbf{x})=\sum_{i=1}^{L} \boldsymbol{\beta}_{i} h_{i}(\mathbf{x})=\mathbf{h}(\mathbf{x}) \boldsymbol{\beta}
$$

where $\boldsymbol{\beta}=\left[\boldsymbol{\beta}_{1}, \ldots, \boldsymbol{\beta}_{L}\right]$ is the output weights between the hidden nodes to output nodes. $\mathbf{h}(\mathbf{x})=\left[h_{1}(\mathbf{x}), \ldots, h_{L}(\mathbf{x})\right]$ is the output vector of the hidden layer with respect to the input $\mathbf{x} ; h_{i}(\mathbf{x})=g\left(\mathbf{w}_{i}, b_{i}, \mathbf{x}\right), \mathbf{w}_{i} \in \mathbf{R}^{d}, b_{i} \in R$ is the output of the $i$-th random hidden node where the hidden node parameters (e.g. w and $b$ ) need not be tuned, in particular, can be randomly generated according to any continuous sampling distribution probability. $g\left(\mathbf{w}_{i}, b_{i}, \mathbf{x}\right)$ is a nonlinear piecewise continuous function satisfying ELM universal approximation capability theorems[16]. For example, such nonlinear piecewise continuous function can be but are not limited to:

1) Sigmoid function

$$
g(\mathbf{w}, b, \mathbf{x})=\frac{1}{1+\exp (-\lambda(\mathbf{w} \cdot \mathbf{x}+b))}
$$

2) Fourier function

$$
g(\mathbf{w}, b, \mathbf{x})=\sin (\mathbf{w} \cdot \mathbf{x}+b)
$$

3) Hardlimit function

$$
g(\mathbf{w}, b, \mathbf{x})= \begin{cases}1 & \text { if } \mathbf{w} \cdot \mathbf{x}-b \geq 0 \\ 0 & \text { otherwise }\end{cases}
$$

4) Gaussian function

$$
g(\mathbf{w}, b, \mathbf{x})=\exp \left(-b\|\mathbf{x}-\mathbf{w}\|^{2}\right)
$$

ELM theory[13, 17, 16, 42] shows that such $\mathbf{h}(\mathbf{x})$ has universal approximation capability, that is, $\lim _{L \rightarrow \infty}\|\mathbf{h}(\mathbf{x}) \boldsymbol{\beta}-f(\mathbf{x})\|_{F}^{2}=\lim _{L \rightarrow \infty}\left\|\sum_{i=1}^{L} \boldsymbol{\beta}_{i} h_{i}(\mathbf{x})-f(\mathbf{x})\right\|_{F}^{2}=0$ hold$\mathrm{s}$ with a probability one for appropriate output weights $\boldsymbol{\beta}$. Given training samples $\boldsymbol{\Omega}=\left\{\left(\mathbf{x}_{i}, \mathbf{t}_{i}\right)\right\}_{i=1}^{N}$, ELM theory aims to reach the smallest norm $\min _{\boldsymbol{\beta}}\|\boldsymbol{\beta}\|_{F}^{2}$ of output weights and training error $\min _{\boldsymbol{\beta}}\|\mathbf{H} \boldsymbol{\beta}-\mathbf{T}\|_{F}^{2}$ where $\mathbf{H}$ is the hidden layer output 
matrix (randomized matrix):

$$
\mathbf{H}=\left[\begin{array}{ccc}
g\left(\mathbf{w}_{1}, b_{1}, \mathbf{x}_{1}\right) & \cdots & g\left(\mathbf{w}_{L}, b_{L}, \mathbf{x}_{1}\right) \\
\vdots & \ddots & \vdots \\
g\left(\mathbf{w}_{1}, b_{1}, \mathbf{x}_{N}\right) & \cdots & g\left(\mathbf{w}_{L}, b_{L}, \mathbf{x}_{N}\right)
\end{array}\right]
$$

and

$$
\mathbf{T}=\left[\begin{array}{c}
\mathbf{t}_{1} \\
\vdots \\
\mathbf{t}_{N}
\end{array}\right]=\left[\begin{array}{ccc}
t_{11} & \cdots & t_{1 m} \\
\vdots & \ddots & \vdots \\
t_{N 1} & \cdots & t_{N m}
\end{array}\right]
$$

$\mathbf{h}(\mathbf{x})$ actually maps the data from the $d$-dimensional input space to the $L$-dimensional hidden layer random feature space. As a special case of $g(\mathbf{w}, b, \mathbf{x}), \mathbf{H}$ can be written as:

$$
\mathbf{H}=g\left([\mathbf{X}, \mathbf{1}]\left[\begin{array}{l}
\mathbf{W} \\
\mathbf{b}
\end{array}\right]\right)=\left[\begin{array}{ccc}
g\left(\mathbf{w}_{1} \cdot \mathbf{x}_{1}+b_{1}\right) & \cdots & g\left(\mathbf{w}_{L} \cdot \mathbf{x}_{1}+b_{L}\right) \\
\vdots & \ddots & \vdots \\
g\left(\mathbf{w}_{1} \cdot \mathbf{x}_{N}+b_{1}\right) & \cdots & g\left(\mathbf{w}_{L} \cdot \mathbf{x}_{N}+b_{L}\right)
\end{array}\right]
$$

Numerous methods are now available for attaining the weight vector $\boldsymbol{\beta}$, such as the No-Prop algorithm suggested by Widrow et al. [43] and the iterative algorithm developed in [44]. A popular and effective solution is given as follows:

$$
\boldsymbol{\beta}=\mathbf{H}^{\dagger} \mathbf{T}
$$

where $\mathbf{H}^{\dagger}$ is the Moore-Penrose generalized inverse of $\mathbf{H}$.

Recently, the unified ELM was introduced in [17, 42], in which $\beta$ is solved as a constrained optimization problem:

$$
\operatorname{Minimize}_{\boldsymbol{\beta}}:\|\mathbf{H} \boldsymbol{\beta}-\mathbf{T}\|_{p}^{\alpha_{1}}+\frac{1}{\gamma}\|\boldsymbol{\beta}\|_{q}^{\alpha_{2}}
$$

where $\alpha_{1}>0, \alpha_{2}>0, p, q=0, \frac{1}{2}, 1,2, \ldots, F,+\infty$ and $\gamma$ is control parameter for the tradeoff of structural risk and experimental risk. Numerous efficient methods can be used to calculate the output weights $\boldsymbol{\beta}$ including but not limited to orthogonal projection method, iterative methods[44], and eigenvalue decomposition method[45]. When 
$p, q=F$ and $\alpha_{1}, \alpha=2$, a popular and efficient closed-form solution[17] is:

$$
\boldsymbol{\beta}= \begin{cases}\mathbf{H}^{\top}\left(\frac{\mathbf{I}}{\gamma}+\mathbf{H} \mathbf{H}^{\top}\right)^{-1} \mathbf{T} & N \geq L \\ \left(\frac{\mathbf{I}}{\gamma}+\mathbf{H}^{\top} \mathbf{H}\right)^{-1} \mathbf{H}^{\top} \mathbf{T} & N \leq L\end{cases}
$$

\subsection{Large-scale Data Analytic}

To process increasing amounts of data efficiently, many efficient and effective algorithms have been proposed. One kind of alternative methods directly avoids expensive computation burden of nonlinear decision functions, and builds large-scale linear algorithms on original feature space such as [46, 47, 48, 49, 50, 51, 52, 53, 54]. This kind of algorithms has showcase competitive generalized performance at fast learning speed in some certain applications such as sparse high dimensional linear separated (or nearly) problems. On the other hand, arising number of researchers are devoting their time and efforts in variants of nonlinear kernel approximation [55, 56, 57] to overcome the challenge of large scale learning. For instances, AMM[55] captures non-linearity by assigning a number of linear hyperplanes to each of classes, and then learned via Stochastic Gradient Descent (SGD). LLSVM[57] approximates kernel SVM optimization by a linear SVM using low-rank decomposition of the kernel matrix. BSGDSVM[56] maintains a fixed number of support vectors in the model, and incrementally updates them during the SGD training. Hsieh etc.[58] proposed a divide-and-conquer solver for kernel SVMs (DC-SVM) where the kernel SVM problem is partitioned into smaller subproblems by clustering the data, so that each subproblem can be solved independently. Random Kitchen Sinks algorithm[59] approximates kernel expansions by means of multiplying the input with a Gaussian random matrix, followed by the application of a nonlinearity. From a survey of the literature [60], the core challenges of Big Data have been widely established and can be summarized under the popular 5 Vs, namely, Volume, Velocity, Variety, Veracity and Value. The present work takes special interests on the volume aspect of Big data by focusing on the massive amounts of data that have been generated across a wide range of sources. In particular, within the context of Big data analytics, volume can be defined by the product of instance size and dimensionality of the data. Taking this cue, in the rest of this section, we present the essential ingredients of our proposed Singular Value Decomposition-Hidden-nodes 
based Extreme Learning Machine, which is designed to work with Big dimensional \&

\section{Proposed Method}

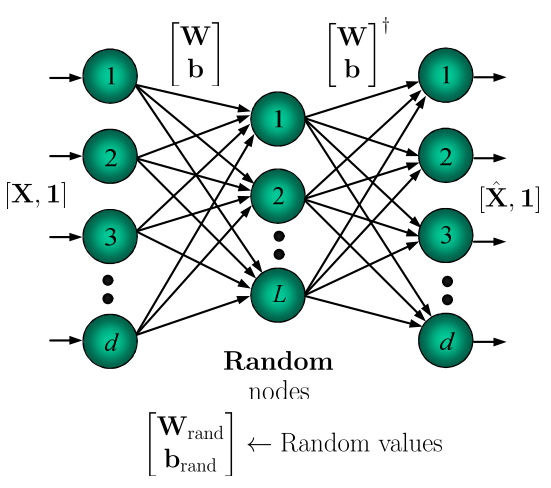

(a) Random hidden nodes

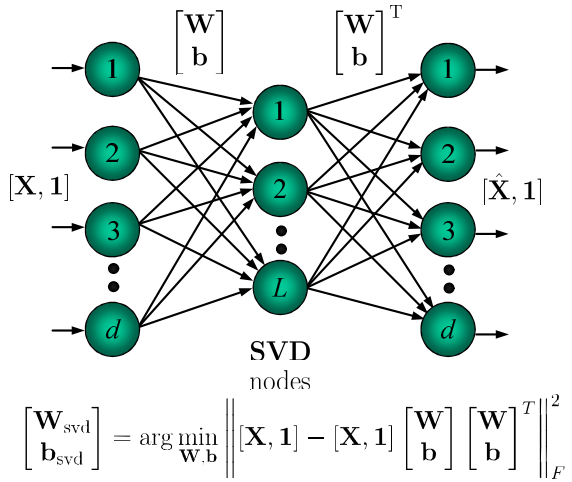

(b) SVD hidden nodes

Figure 1: Random hidden nodes in classical ELM and SVD hidden nodes in our proposed method. For random hidden nodes, the parameters of the hidden nodes are randomly generated according to some continuous sampling distribution probability, while for SVD hidden nodes, the parameters of the hidden nodes are generated under the conditions of optimal low rank approximation.

\subsection{Problem of Interests}

Given training set $\boldsymbol{\Omega}=\left\{\left(\mathbf{x}_{i}, \mathbf{t}_{i}\right) \mid \mathbf{x}_{i} \in \mathbf{R}^{d}, \mathbf{t}_{i} \in \mathbb{R}^{m}\right\}_{i=1}^{N}$, where $d$ is the data dimension, $N$ is instance size and $m$ is the number of outputs. In a single-output regression problem, $\mathbf{t}_{i} \in R$ is a continuous real value $(\mathbb{R}=R)$. For a $m$-class classification problem, $\mathbf{t}_{i} \in\{0,1\}^{m}$ is a $m$-dimensional boolean vector $(\mathbb{R}=\{0,1\})$. If the original class label is $p$, the expected output vector of the $m$ outputs is $\mathbf{t}_{i}=[0, \ldots, 0, \stackrel{p}{1}, 0, \ldots, 0]_{1 \times m}$. In this case, only the $p$-th element of $\mathbf{t}_{i}=\left[t_{1}, t_{2}, \ldots, t_{m}\right]$ is ' 1 ', while the rest of the elements are set to ' 0 '. Binary classification is thus considered a special case of the multi-class problem where $m=2$.

In the current work, our core interest is in the case where $N$ and $d$ are 'Big'. On Big instance data, where only $N$ is big, many effective algorithms has been proposed and 
studied [61, 55, 62, 63, 64, 65]. However, despite the many ongoing efforts, majority (78.8\%) [1] of the studies on Big data analytic have been confined to Big instance data with features that are not so 'Big', i.e., typically lower than 100, 000 in dimensions. On Big instance and Big dimensional data, both $N$ and $d$ are very large, and to date less than $4 \%[1]$ of the studies in the computational intelligence community have considered real world data with features that range in the millions [66, 67]. Thus, it is clear that the dimensionality of the algorithms under-studied is significantly lagging behind those being produced, mainly due to the many great challenges of Big dimensional and Big instance data.

\subsection{Fast SVD-Hidden-Nodes Based Extreme Learning Machine for Big Dimensional} \& Big Instance Data

By treating the hidden layer of ELM as unsupervised generative model[68] with $L$ hidden nodes, it plays the role of learning more useful (or discriminative) features from original data without losing much information. When the number of hidden nodes $L$ is equal to or greater than the data dimensions, i.e., $L \geq d$, the input weights could be any unitary matrices to make reconstruction error approximating to zero, and thus it will become a trivial matrix; but by placing the constraint on the network by limiting the number of hidden nodes, we can learn essential embedding structure of the data. In special, the hidden layer as generative model is to re-represent the data with minimal reconstruction error $\left\|\left[\begin{array}{ll}\mathbf{X} & 1\end{array}\right]-\left[\begin{array}{ll}\hat{\mathbf{X}} & \mathbf{1}\end{array}\right]\right\|_{F}^{2}$ and minimal hidden nodes $L$, that is:

$$
\operatorname{Minimize}_{\mathbf{W}, \mathbf{b}}:\left\|\left[\begin{array}{ll}
\mathbf{X} & \mathbf{1}
\end{array}\right]-\left[\begin{array}{ll}
\mathbf{X} & \mathbf{1}
\end{array}\right]\left[\begin{array}{l}
\mathbf{W} \\
\mathbf{b}
\end{array}\right]\left[\begin{array}{l}
\mathbf{W} \\
\mathbf{b}
\end{array}\right]^{\top}\right\|_{F}^{2}
$$

where $\mathbf{W} \in \mathbf{R}^{d \times L}$ and $\mathbf{b} \in \mathbf{R}^{1 \times L}$ are input weights and bias, $[\hat{\mathbf{X}} \mathbf{1}]$ is the rerepresentation in $L$-dimension feature space of the original data.

In the classical ELM implementation, the above optimization condition is not considered and the hidden nodes have been randomly generated:

$$
\left[\begin{array}{c}
\mathbf{W}_{\text {rand }} \\
\mathbf{b}_{\text {rand }}
\end{array}\right] \leftarrow \text { Random values }
$$




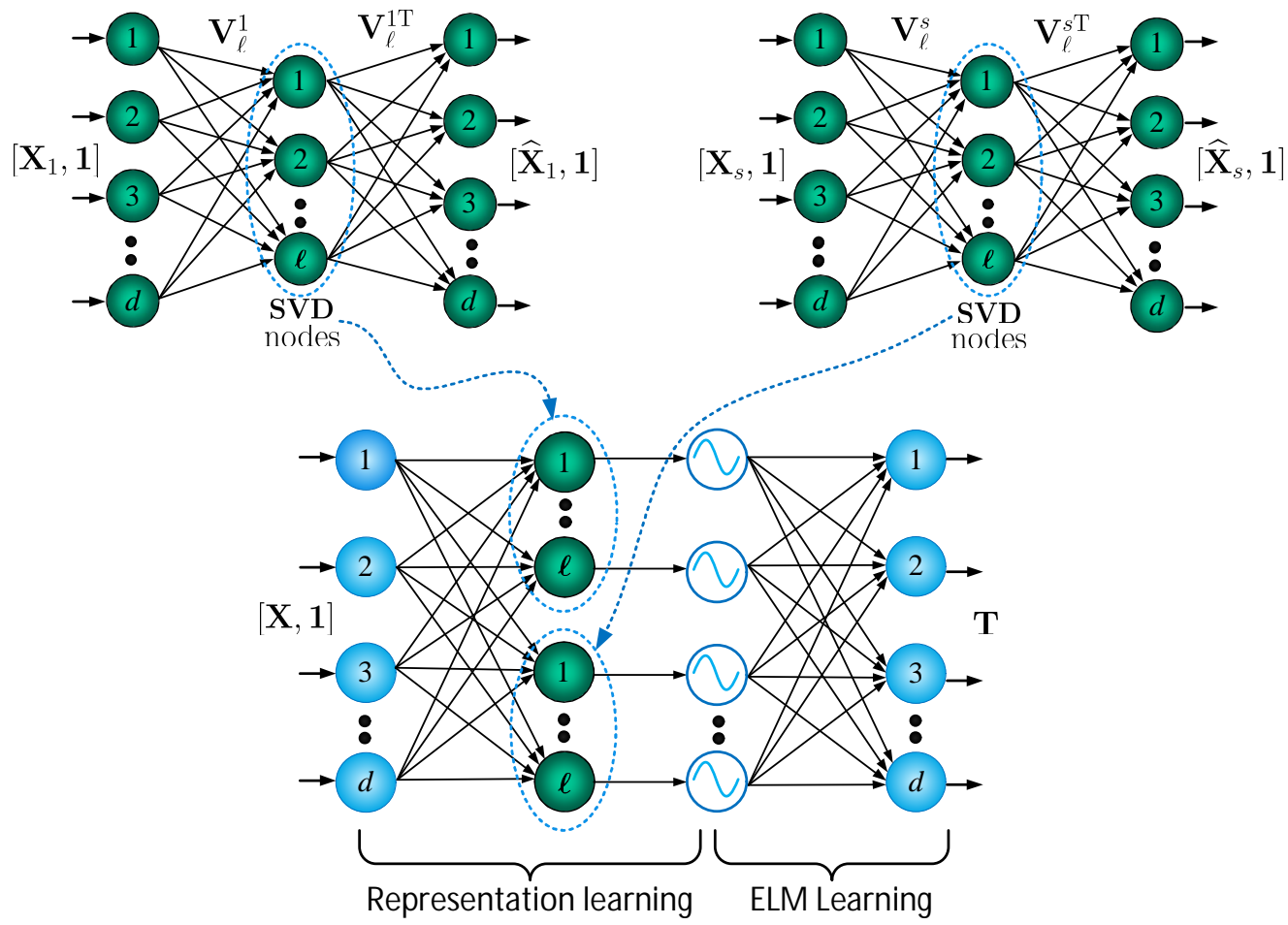

Figure 2: Fast divide-and-conquer approximation scheme for Big data (Big instance size and Big dimensionality). In the divide-and-conquer scheme, the complete data set is first sliced into multiple small subsets without overlapping according to a uniform distribution, and then each subset generates one part of the hidden nodes for FSVD-H-ELM such that reconstruction error is minimized independently.

The random hidden nodes architecture of the classical ELM is depicted in Fig 1.(a), which provides a simple approach for fast learning. However, when faced with Big dimensional data, the use of random hidden nodes tend to suffer since they fail to capture the true intrinsic characteristics of the Big dimensionality, resulting in a poor representation of the data in the hidden layer. Traditionally, researchers would use Principal Components Analysis (PCA) and other dimensionality reduction methods to work on the Big dimensional data as a form of pre-processing before building the classifiers [69, 3, 70, 71, 72]. However, such a scheme would require additional memory space for archiving the preprocessed data[73]. This can pose as an issue when dealing with 
Big data. In contrast to previous studies in the literature, we embed hidden nodes that are designed using Singular Value Decomposition (SVD) directly into the classical ELM to arrive at the proposed SVD-H-ELM. Particularly, we generate the SVD hidden nodes based on the optimal rank- $L$ SVD as shown in Fig 1-(b). As proved in the following, SVD hidden nodes can satisfy the optimization condition of Eq.99.

We consider the SVD of $[\mathbf{X}, \mathbf{1}]$ as

$$
[\mathbf{X}, \mathbf{1}] \stackrel{\text { SVD }}{=} \mathbf{U S V}^{\top}
$$

where $\mathbf{U} \in \mathbf{R}^{N \times N}$ and $\mathbf{V} \in \mathbf{R}^{d \times d}$ are unitary matrices, and $\mathbf{S} \in \mathbf{R}^{N \times d}$ is diagonal matrix with non-negative real numbers with decent order $\sigma_{1}>\sigma_{2}>, \ldots,>\sigma_{N}$ on the diagonal.

The optimal rank- $L$ approximation of $[\mathbf{X}, \mathbf{1}]$ is given by zeroing out the $\min \{N, d\}$ $L$ trailing values of $\mathbf{S}$, that is:

$$
[\mathbf{X}, \mathbf{1}] \triangleq \mathbf{U}_{L} \mathbf{S}_{L} \mathbf{V}_{L}^{\top}
$$

where $\mathbf{U}_{L}$ and $\mathbf{V}_{L}$ denote the first $L<\min \{N, d\}$ columns of $\mathbf{U}$ and $\mathbf{V}$, and $\mathbf{S}_{L}=$ $\operatorname{diag}\left(\sigma_{1}, \sigma_{2}, \ldots, \sigma_{L}\right)$.

Parameter of the hidden nodes can then be obtained as

$$
\left[\begin{array}{c}
\mathbf{W}_{\text {SVD }} \\
\mathbf{b}_{\text {SVD }}
\end{array}\right] \leftarrow \mathbf{V}_{L}
$$

By replacing the random parameter of the hidden nodes with $\left(\mathbf{W}_{\mathrm{SVD}}, \mathbf{b}_{\mathrm{SVD}}\right)$ and incorporating them as part of the ELM network thus arrive at the proposed SVD-HELM network. Consequently, the latent properties of the data can be appropriately represented via the SVD hidden nodes of the SVD-H-ELM.

Particularly, ( $\left.\mathbf{W}_{\mathrm{SVD}}, \mathbf{b}_{\mathrm{SVD}}\right)$ gives the minimal reconstruction error with Forbenius norm according to the following theorem:

Theorem 1. The SVD nodes with parameter $\left(\mathbf{W}_{S V D}, \mathbf{b}_{S V D}\right)$ can provide more accurate representation of input $\mathbf{X}$ in the measure of Forbenius norm than random nodes with $\left(\mathbf{W}_{\text {rand }}, \mathbf{b}_{\text {rand }}\right)$.

Proof. Suppose that we have a classical ELM, in which parameter of the hidden nodes $\left(\mathbf{W}_{\text {Rand }}, \mathbf{b}_{\text {Rand }}\right)$ is randomly generated according to any continuous sampling distribu- 
tion probability, the Forbenius norm reconstruction error of the original data can be derived as:

$$
\xi_{\text {Rand }}=\left\|[\mathbf{X}, \mathbf{1}]-[\mathbf{X}, \mathbf{1}]\left[\begin{array}{c}
\mathbf{W}_{\text {Rand }} \\
\mathbf{b}_{\text {Rand }}
\end{array}\right]\left[\begin{array}{c}
\mathbf{W}_{\text {Rand }} \\
\mathbf{b}_{\text {Rand }}
\end{array}\right]^{\dagger}\right\|_{F}^{2}
$$

For the SVD hidden nodes in SVD-H-ELM where the parameter of hidden nodes are generated as 13 , the reconstruction error of the original data is given:

$$
\xi_{\mathrm{SVD}}=\left\|[\mathbf{X}, \mathbf{1}]-[\mathbf{X}, \mathbf{1}]\left[\begin{array}{c}
\mathbf{W}_{\mathrm{SVD}} \\
\mathbf{b}_{\mathrm{SVD}}
\end{array}\right]\left[\begin{array}{c}
\mathbf{W}_{\mathrm{SVD}} \\
\mathbf{b}_{\mathrm{SVD}}
\end{array}\right]^{\top}\right\|_{F}^{2}=\left\|[\mathbf{X}, \mathbf{1}]-\mathbf{U}_{L} \mathbf{S}_{L} \mathbf{V}_{L}^{T}\right\|_{F}^{2}
$$

where $\mathbf{U}_{L} \mathbf{S}_{L} \mathbf{V}_{L}^{T}$ is optimal rank- $L$ approximation of $[\mathbf{X}, \mathbf{1}]$ as shown in (12).

According to the Eckart-Young-Mirsky theorem [74], the rank- $L$ approximation with the $L$ largest singular values is the solution with the least reconstruction error, that is $\xi_{\text {SVD }}<\xi_{\text {Rand }}$ always holds. This means that SVD nodes yield more accurate representation of input $\mathbf{X}$ than random nodes in the hidden layer.

Remark 1: In practice, noise often exists in the data. Assume that the noise is i.i.d. Gaussian noise. When dealing with data of low-to-medium dimensionality, the noise does not pose much of an issue [69]. However, when dealing with data of Big dimensionality, the noise generally dominates in the last few principal components with smallest singular values, because the i.i.d. Gaussian noise is invariant across all principal components transformed by the input weight $\left[\mathbf{W}_{\mathrm{SVD}}^{\top}, \mathbf{b}_{\mathrm{SVD}}^{\top}\right]^{\top}=\mathbf{V}_{L}$, while the true signal variance is small in the last few components. In this case, the SVD hidden nodes in SVD-H-ELM provide more meaningful representations of the input $\mathbf{X}$ than random hidden nodes by disposing the last few components. The above thus summarizes the theoretical proof and analysis on the virtue of using SVD hidden nodes in SVD-H-ELM over the use of random hidden nodes in classic ELM.

\subsection{A Fast Divide \& Conquer Approximation Scheme}

With the availability of SVD hidden nodes, improved representations of the Big dimensional data can be achieved in the ELM over using random hidden nodes, thus 
enabling the proposed SVD-H-ELM to attain improved generalization performance and stability. The drawback of SVD-H-ELM, however, is the high computational complexity of SVD, especially when working with Big instance problems. Therefore, in this subsection, we present a fast approximation scheme as shown in Fig 2 , which takes inspiration from the concept of divide and conquer, to achieve computational tractability on large-scale problems. The proposed algorithm is then labelled here as the Fast Singular Value Decomposition-Hidden nodes-Extreme Learning Machine or FSVDH-ELM. In FSVD-H-ELM, instead of identifying the SVD hidden nodes directly from the whole data, SVD hidden nodes are derived from multiple random subsets of data sampled from the original data, thus achieving improved scalability on Big instance and Big dimensional problems.

To date, many different methods to approximate the SVD at improved asymptotic running time have been described[75][76]For instance, in [75], the decomposition was performed using $\mathrm{QR}$ to a random embedding matrix $\mathbf{C}=\mathbf{X M}$, in which $\mathbf{M} \in \mathbf{R}^{d \times k}(k<d)$ is a random matrix and $\mathbf{X} \in \mathbf{R}^{N \times d}$ is the data matrix. The total computation burden is around $\mathcal{O}\left(N d k+N^{2} k\right)$. In [76], sparsification was used on the data matrix by retaining an element $x_{i j}$ with probability $p_{i j}$ or replacing it with 0 otherwise. Although these approximation approaches can improve decomposition efficiency, since for Big instances and Big dimension data, the instances size $N$ and and the dimensions $d$ are both very large, the approximate complexity is still considerable expensive. To tackle this problem, we adopt an efficient sampling scheme, where multiple subsets are constructed by picking random samples from the original data. The SVD hidden nodes derived from each of the multiple data subsets then serve as the approximation or generalization of the original data. Our scheme provides good approximation to the SVD of the original dataset with a bounded error. Intuitively, if the sampling is fair, these subsets can capture the general distribution of the data. In Section IV, theoretical proof and analysis were presented for our method. In summary, the SVD hidden nodes derived from these subsets can generally produce good representation of the Big dimensional data, while requiring tractable computational effort.

In our divide-and-conquer scheme, the complete data is first sliced (or divided) into $S=\left\lfloor\frac{N}{n}\right\rfloor$ subsets without overlapping such that each subset has around $n$ samples. 
Each subset produces $\ell=\rho \cdot n$ hidden nodes where $0<\rho<1$ is the ratio of the retained rank, then $s$ subsets $\left\{\mathbf{X}_{r}\right\}_{r=1}^{s}$ are randomly selected from $S$ subsets such that $L=\sum_{r=1}^{s} \ell$ where $L$ is the total number of hidden nodes in FSVD-H-ELM.

For each data subset in $\left\{\mathbf{X}_{r}\right\}_{r=1}^{s}$, the optimal rank- $\ell$ approximation is obtained similar to (12):

$$
\left[\mathbf{X}_{r}, \mathbf{1}\right] \triangleq \mathbf{U}_{\ell}^{r} \mathbf{S}_{\ell}^{r} \mathbf{V}_{\ell}^{r T}
$$

Simply assigning the parameter of $\ell$ hidden nodes, i.e. $\{(r-1) \cdot \ell+1,(r-1) \cdot \ell+2, \ldots, r \cdot \ell\}-$ th superscript nodes with $\mathbf{V}_{\ell}^{r}$ and concatenating them together, then the parameter of the $L$ hidden nodes can be obtained as following

$$
\left[\begin{array}{c}
\mathbf{W}_{\mathrm{FSVD}} \\
\mathbf{b}_{\mathrm{FSVD}}
\end{array}\right] \Leftarrow\left[\begin{array}{c:c:c:c}
\mathbf{W}_{1} & \mathbf{W}_{2} & \ldots & \mathbf{W}_{s} \\
\mathbf{b}_{1} & \mathbf{b}_{2} & \mathbf{b}_{s}
\end{array}\right] \Leftarrow\left[\mathbf{V}_{\ell}^{1}\left|\mathbf{V}_{\ell}^{2}\right| \ldots \mid \mathbf{V}_{\ell}^{s}\right]
$$

Therefore, the hidden layer output matrix of SVD hidden nodes can be derived as:

$$
\begin{aligned}
& \mathbb{P}_{L}=[\mathbf{X}, \mathbf{1}]\left[\begin{array}{c}
\mathbf{W}_{\mathrm{FSVD}} \\
\mathbf{b}_{\mathrm{FSVD}}
\end{array}\right]=[\mathbf{X}, \mathbf{1}]\left[\begin{array}{lll}
\mathbf{V}_{\ell}^{1} & \cdots & \mathbf{V}_{\ell}^{s}
\end{array}\right] \\
& \mathbf{H}=g\left(\mathbb{P}_{L}\right)=g\left(\mathbf{X}\left[\begin{array}{lll}
\mathbf{V}_{\ell}^{1} & \cdots & \mathbf{V}_{\ell}^{s}
\end{array}\right]\right)
\end{aligned}
$$

\subsection{Computational Complexity}

Here we analyze the computational complexities of the classical SVD (used in SVD-H-ELM) and our proposed divide-and-conquer SVD approximation scheme (used in FSVD-H-ELM). In particular, since the complexity of SVD on a $r \times c$ input data matrix is $\mathbf{X} \mathcal{O}(r c \cdot \min (r, c))$, it can be easily derived that:

$$
\mathrm{O}_{\text {classical-SVD }}=\mathcal{O}(N d \cdot \min (N, d))
$$

and

$$
\mathrm{O}_{\text {Divide \& Conquer Approx-SVD }}=\mathcal{O}\left(\sum_{r=1}^{s} n^{2} d\right)
$$

Further, since the subset size $n$ is typically set to be small $(n \ll N)$ for the purpose of efficiency, it can be easily observed that the computational complexity of the fast divideand-conquer approximation scheme $\mathrm{O}_{\text {Divide \& Conquer Approx-SvD }}$ is much lower than that of the classical SVD $O_{\text {classical-SVD. }}$. 
Remark 2: Since the SVD of each subset $\mathbf{X}_{r}, r=1,2, \ldots, s$ is conducted independently, parallel computing of the scheme can be conveniently realized. Therefore, the memory requirement is significantly reduced and the training speed can also be greatly improved.

Remark 3: The proposed algorithm is also suitable for online model update than other two-stage algorithms, where the latter involves dimensionality reduction as a pre-processing stage before the learning stage. For any two-stage algorithm, with the arrival of a new data point, besides the mapping matrix of dimensionality reduction that needs to be performed again in order to update, it also requires the learning algorithms to be recomputed or rebuilt. In the context of ELM, the number of hidden nodes $L$ would need to be revised accordingly each time some new data points are received. On the contrary, with our proposed algorithm, $L$ is the number of the retained components or the rank, i.e., $\sum_{r=1}^{s} \ell$. Thus, the value of $L$ can be naturally adjusted by the rank- $\ell$ online updating in the online phase. Furthermore, with our scheme, there is no need for additional memory to store the preprocessed data after performing dimensionality reduction.

\section{A Discussion on the Feature Learning Ability of the Fast Divide \& Conquer Approximation Scheme}

In this section, we present an analysis on the Frobenius reconstruction error bound of the fast divide-and-conquer approximation scheme and compares it to the classical SVD on the original complete dataset.

First, we define the following notations which are used in the analysis:

- $\mathbb{P}_{L}=[\mathbf{X}, \mathbf{1}]\left[\mathbf{V}_{\ell}^{1} \cdots \mathbf{V}_{\ell}^{s}\right]$ : The $L$-dimensional representation of the original dataset (see (18)) in the divide-and-conquer scheme with $s$ subsets $\left\{\mathbf{X}_{r}\right\}_{r=1}^{s}$;

- $\mathbb{P}_{\ell}^{r}=[\mathbf{X}, \mathbf{1}]\left[\mathbf{V}_{\ell}^{r}\right]$ : The $\ell$-dimensional representation of the original dataset in the divide-and-conquer scheme with single subset $\mathbf{X}_{r}$;

- $\mathbf{P}_{\ell}=[\mathbf{X}, \mathbf{1}]\left[\mathbf{V}_{\ell}\right]$ : The $\ell$-dimensional representation of the original dataset in the optimal classical rank- $\ell$ SVD; 


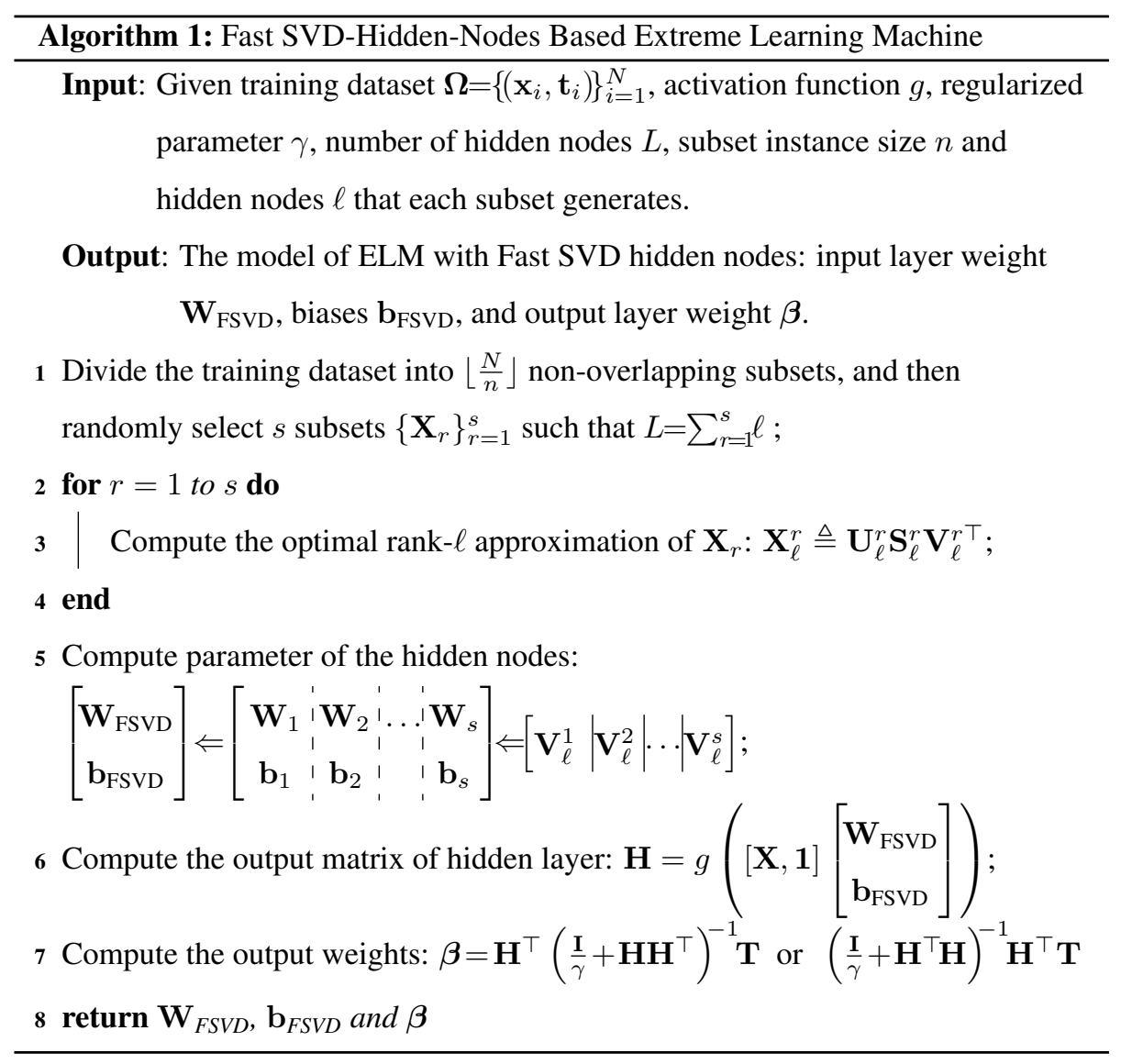


Theorem 2. Suppose $N$ samples $\mathbf{X}=\left\{\mathbf{x}_{i} \mid \mathbf{x}_{i} \in \mathbf{R}^{m}\right\}_{i=1}^{N}$ are divided into $\left\lfloor\frac{N}{n}\right\rfloor$ subsets with subset size $n$, and each subset produces $\ell$ hidden nodes. The s subsets $\left\{\mathbf{X}_{r}\right\}_{r=1}^{s}$ are then randomly selected such that the $L=\sum_{r=1}^{s}$ l where $L$ is the total number of hidden nodes in FSVD-H-ELM. Then with probability of at least $(1-\delta)$, the following inequality holds:

$\left\|\mathbf{X X}^{\top}-\mathbb{P}_{L} \mathbb{P}_{L}^{\top}\right\|_{F} \leq\left\|\mathbf{X X}^{\top}-\mathbf{P}_{\ell} \mathbf{P}_{\ell}^{\top}\right\|_{F}+N \theta_{D}\left(\frac{64 \ell}{n}\right)^{\frac{1}{4}}\left\{1+\left[\frac{2(N-s n) \theta_{d i s}^{2} \log \frac{1}{\delta}}{s\left(N-\frac{1}{2}\right)\left(1-\frac{1}{2} \max \{s n, N-s n\}\right)}\right]^{\frac{1}{2}}\right\}^{\frac{1}{2}}$

where

$\theta_{D}=\max _{i}\left(\mathbf{x}_{i} \mathbf{x}_{i}^{\top}\right)^{\frac{1}{2}}$ and $\quad \theta_{d i s}=\max _{i, j}\left(\mathbf{x}_{i} \mathbf{x}_{i}^{\top}+\mathbf{x}_{j} \mathbf{x}_{j}^{\top}-2 \mathbf{x}_{i} \mathbf{x}_{j}^{\top}\right)^{\frac{1}{2}}$.

Proof. For $r \in[1, s]$, let $\mathbf{Z}_{r}=\sqrt{N / b} \mathbf{X} \mathcal{S}_{r}$, where $\mathcal{S}_{r}$ denotes the selection matrix corresponding to the subset $\mathbf{X}_{r}$. By definition of $\mathbb{P}_{L}$, the following condition holds:

$$
\left\|\mathbf{X X}^{\top}-\mathbb{P}_{L} \mathbb{P}_{L}^{\top}\right\|_{F}^{2} \leq \frac{1}{s}\left\|\sum_{i=r}^{s} \mathbf{X} \mathbf{X}^{\top}-\mathbb{P}_{\ell}^{r} \mathbb{P}_{\ell}^{r T}\right\|_{F}^{2} \leq \frac{1}{s} \sum_{i=r}^{s}\left\|\mathbf{X} \mathbf{X}^{\top}-\mathbb{P}_{\ell}^{r} \mathbb{P}_{\ell}^{r T}\right\|_{F}^{2}
$$

Note that $\mathbb{P}_{\ell}^{r} \mathbb{P}_{\ell}^{r T} \approx\left(\mathbf{X X}_{r}^{\top}\right)\left(\mathbf{X}_{r} \mathbf{X}_{r}\right)^{-1}\left(\mathbf{X}_{r} \mathbf{X}^{\top}\right)$ is equivalent to the standard Nyström approximation of $\mathbf{X X}^{\top}$. Hence, the following general inequality holds for the Frobenius error of the standard Nyström approximation method [5]:

$$
\left\|\mathbf{X X}^{\top}-\mathbb{P}_{\ell}^{r} \mathbb{P}_{\ell}^{r T}\right\|_{F}^{2} \leq\left\|\mathbf{X X}^{\top}-\mathbf{P}_{\ell} \mathbf{P}_{\ell}^{\top}\right\|_{F}^{2}+N \theta_{\mathrm{D}} \sqrt{64 \ell}\left\|\mathbf{X X}^{\top}-\mathbf{Z}_{r} \mathbf{Z}_{r}^{\top}\right\|_{F}
$$

where $\left\|\mathbf{X X}^{\top}-\mathbf{P}_{\ell} \mathbf{P}_{\ell}^{\top}\right\|_{F}^{2}$ is the Frobenius error bound of the classical SVD with reduced rank- $\ell$. Substituting 23 , into [22], we have:

$$
\left\|\mathbf{X} \mathbf{X}^{\top}-\mathbb{P}_{L} \mathbf{P}_{L}^{\top}\right\|_{F}^{2} \leq\left\|\mathbf{X} \mathbf{X}^{\top}-\mathbf{P}_{\ell} \mathbf{P}_{\ell}^{\top}\right\|_{F}^{2}+\frac{N \theta_{\mathrm{D}} \sqrt{64 \ell}}{s} \sum_{i=r}^{s}\left\|\mathbf{X X}^{\top}-\mathbf{Z}_{r} \mathbf{Z}_{r}^{\top}\right\|_{F}
$$

Denote

$$
\phi\left(\mathcal{S}_{r}\right)=\frac{1}{s} \sum_{r=1}^{s}\left\|\mathbf{X} \mathbf{X}^{\top}-\mathbf{Z}_{r} \mathbf{Z}_{r}^{\top}\right\|_{F}
$$

Let $\mathcal{S}_{r}^{\prime}$ be a sampling matrix that comprises the same columns as $\mathcal{S}_{r}$ except for one, and let $\mathbf{Z}_{r}^{\prime}$ denote $\sqrt{N / b} \mathbf{X} \mathcal{S}_{r}^{\prime}$. Changing one column of the full sample $\mathcal{S}$ (the selection 
matrix corresponding to $\left\{\mathbf{X}_{r}\right\}_{r=1}^{s}$ ) changes only one subsample $\mathcal{S}_{r}$ and thus only the single term $\left\|\mathbf{X} \mathbf{X}^{\top}-\mathbf{Z}_{r} \mathbf{Z}_{r}^{\top}\right\|_{F}$. Let $\mathbf{z}$ and $\mathbf{z}^{\prime}$ denote the only differing column of $\mathbf{Z}_{r}$ and $\mathbf{Z}_{r}^{\prime}$, then we have:

$$
\begin{aligned}
& \left|\phi\left(\mathcal{S}_{r}^{\prime}\right)-\phi\left(\mathcal{S}_{r}\right)\right| \leq \frac{1}{s}\left\|\mathbf{z}^{\prime} \mathbf{z}^{\prime \top}-\mathbf{z} \mathbf{z}^{\top}\right\|_{F} \\
& =\frac{1}{s}\left\|\mathbf{z}^{\prime} \mathbf{z}^{\prime \top}-\mathbf{z}^{\prime \top}+\mathbf{z}^{\prime \top}-\mathbf{z z}^{\top}\right\|_{F}=\frac{1}{s}\left\|\left(\mathbf{z}^{\prime}-\mathbf{z}\right) \mathbf{z}^{\prime \top}+\mathbf{z}\left(\mathbf{z}^{\prime \top}-\mathbf{z}^{\top}\right)\right\|_{F} \\
& \leq \frac{2}{s}\left\|\mathbf{z}^{\prime}-\mathbf{z}\right\|_{F} \max \left\{\|\mathbf{z}\|_{F},\left\|\mathbf{z}^{\prime}\right\|_{F}\right\}
\end{aligned}
$$

Thus, the columns of $\mathbf{Z}_{r}$ are those of $\mathbf{X}$ as scaled by $\sqrt{N / n}$. The norm of the difference for two columns of $\mathbf{X}$ can be viewed as the norm of the difference for two feature vectors and thus can be derived to be bounded by $\theta_{\text {dis }}$. Similarly, the norm of a single column of $\mathbf{X}$ is bounded by $\theta_{\mathrm{D}}$. This leads to the following inequality:

$$
\left|\phi\left(\mathcal{S}_{r}^{\prime}\right)-\phi\left(\mathcal{S}_{r}\right)\right| \leq \frac{2 N \theta_{\text {dis }} \theta_{\mathrm{D}}}{s n}
$$

Further, following Corollary 2 of [77], the expectation of $\phi\left(\mathcal{S}_{r}\right)$ can be bounded as follows:

$$
E\left[\phi\left(\mathcal{S}_{r}\right)\right]=\frac{1}{s} \sum_{r=1}^{s} E\left[\left\|\mathbf{X} \mathbf{X}^{\top}-\mathbf{Z}_{r} \mathbf{Z}_{r}^{\top}\right\|_{F}\right] \leq \frac{N \theta_{\mathrm{D}}}{\sqrt{n}}
$$

And following Theorem 1 of [78], we know:

$$
\mathcal{P}\left[\phi\left(\mathcal{S}_{r}\right)-E\left[\phi\left(\mathcal{S}_{r}\right)\right]>\epsilon\right] \leq \exp \left(\frac{-2 \epsilon^{2}}{\alpha(s n, N)\left|\phi\left(\mathcal{S}_{r}\right)-\phi\left(\mathcal{S}_{r}^{\prime}\right)\right|^{2}}\right)
$$

where

$$
\alpha(s n, N)=\frac{s b(N-s n)}{\left(N-\frac{1}{2}\right)(1-1 /(2 \max \{s n, N-s n\}))}
$$

Denote $\delta=\exp \left(\frac{-2 \epsilon^{2}}{\alpha(s n, N)\left|\phi\left(\mathcal{S}_{r}\right)-\phi\left(\mathcal{S}_{r}^{\prime}\right)\right|^{2}}\right)$ then we have:

$$
\log \delta=\frac{-2 \epsilon^{2}}{\alpha(s n, N)\left|\phi\left(\mathcal{S}_{r}\right)-\phi\left(\mathcal{S}_{r}^{\prime}\right)\right|^{2}}
$$

and then we have:

$$
\epsilon=\left[\frac{\alpha(s n, N)}{2}\left|\phi\left(\mathcal{S}_{r}\right)-\phi\left(\mathcal{S}_{r}^{\prime}\right)\right|^{2} \log \frac{1}{\delta}\right]^{\frac{1}{2}}
$$


From the above, it is noted that with probability $\operatorname{Pr}\left[\phi\left(\mathcal{S}_{r}\right)-E\left(\phi\left(\mathcal{S}_{r}\right)\right) \geq \epsilon\right] \leq \delta$, the following condition holds:

$$
\phi\left(\mathcal{S}_{r}\right)-E\left[\phi\left(\mathcal{S}_{r}\right)\right] \geq\left[\frac{\alpha(s n, N)}{2}\left|\phi\left(\mathcal{S}_{r}\right)-\phi\left(\mathcal{S}_{r}^{\prime}\right)\right|^{2} \log \frac{1}{\delta}\right]^{\frac{1}{2}}
$$

then,

$$
\begin{aligned}
\phi\left(\mathcal{S}_{r}\right) & \geq\left[\frac{\alpha(s n, N)}{2}\left|\phi\left(\mathcal{S}_{r}\right)-\phi\left(\mathcal{S}_{r}^{\prime}\right)\right|^{2} \log \frac{1}{\delta}\right]^{\frac{1}{2}}+E\left[\phi\left(\mathcal{S}_{r}\right)\right] \\
& =\left[\frac{\alpha(s n, N)}{2}\left(\frac{2 N \theta_{\text {dis }} \theta_{\mathrm{D}}}{s n}\right)^{2} \log \frac{1}{\delta}\right]^{\frac{1}{2}}+\frac{N \theta_{\mathrm{D}}}{\sqrt{n}}
\end{aligned}
$$

In other words, with at least $(1-\delta)$ probability, the following condition holds:

$$
\phi\left(\mathcal{S}_{r}\right) \leq\left[\frac{\alpha(s n, N)}{2}\left(\frac{2 N \theta_{\text {dis }} \theta_{\mathrm{D}}}{s n}\right)^{2} \log \frac{1}{\delta}\right]^{\frac{1}{2}}+\frac{N \theta_{\mathrm{D}}}{\sqrt{n}}
$$

Combining (24), (25) and (34) we arrive at:

$$
\begin{aligned}
& \left\|\mathbf{X X}^{\top}-\mathbb{P}_{L} \mathbb{P}_{L}^{\top}\right\|_{F}^{2} \leq\left\|\mathbf{X X}^{\top}-\mathbf{P}_{\ell} \mathbf{P}_{\ell}^{\top}\right\|_{F}^{2}+ \\
& N \theta_{\mathrm{D}} \sqrt{64 \ell}\left\{\left[\frac{\alpha(s n, N)}{2}\left(\frac{2 N \theta_{\text {dis }} \theta_{\mathrm{D}}}{s n}\right)^{2} \log \frac{1}{\delta}\right]^{\frac{1}{2}}+\frac{N \theta_{\mathrm{D}}}{\sqrt{n}}\right\}
\end{aligned}
$$

Or

$$
\begin{aligned}
& \left\|\mathbf{X X}^{\top}-\mathbb{P}_{L} \mathbb{P}_{L}^{\top}\right\|_{F} \leq\left\|\mathbf{X X}^{\top}-\mathbf{P}_{\ell} \mathbf{P}_{\ell}^{\top}\right\|_{F}+ \\
& N \theta_{\mathrm{D}}\left(\frac{64 \ell}{n}\right)^{\frac{1}{4}}\left\{1+\left[\frac{2(N-s n) \theta_{\mathrm{dis}}^{2} \log \frac{1}{\delta}}{s\left(N-\frac{1}{2}\right)\left(1-\frac{1}{2} \max \{s n, N-s n\}\right)}\right]^{\frac{1}{2}}\right\}^{\frac{1}{2}}
\end{aligned}
$$
denoising technology have been proven in the literature to learn more useful feature representation[79]. Our experimental results in the next section also verify the hypothesis. 


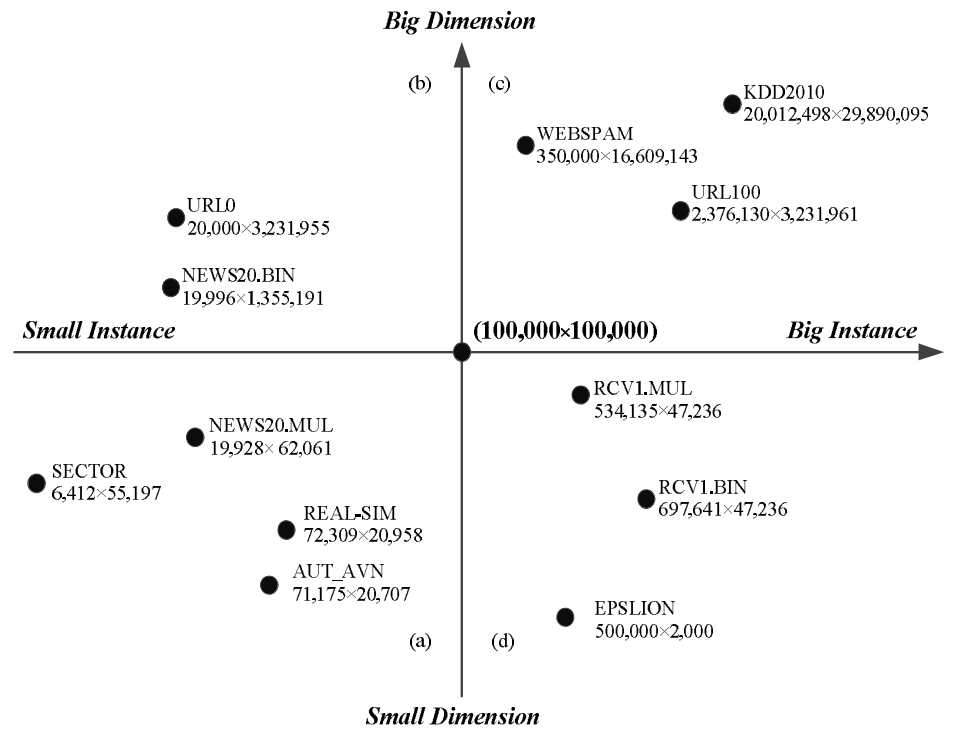

Figure 3: Four groups of data: (a) Small instance \&Small dimension (REAL-S IM, NEWS2 0.MUL, AUT_AVN, SECTOR); (b) Small instance\& Big dimension (URLO, NEWS20.BIN); (c) Big instance \& Big dimension (WEBSPAM, KDD2010, URL100); and (d) Big instance \& Small dimension (RCV1.MUL, RCV1.BIN, EPSLION). $N \times d$ : Instances $\times$ Dimensions. Big dimension: $d \geqslant 100,000$, Big instance size: $N \geqslant 100,000$.

\section{Experimental Study and Performance Evaluation}

In this section, we evaluate the performance of the proposed method, termed FSVD-

H-ELM, and assess it against several state-of-the-art methods on 12 datasets which belong to 4 cases as show in Fig 3 . Among them, all datasets except AUt_AVN 1 are obtained from the LIBSVM website URLO and URL100 are the URL datas from an anonymized 121-day subset of the ICML-09 URL data[80]. The original URL dataset contains 121 independent subsets collected from 121 days. URLO is the data from the first days using day- 0 to predict day- 1 , while URL100 is the data from the first 100 days using them predict the rest days. These datasets have already been split into training and testing set as shown in Table 1

\footnotetext{
1 http://vikas.sindhwani.org/svmlin.html

2 http://www.csie.ntu.edu.tw/ cjlin/libsvmtools/datasets/
} 
All the experiments are conducted on a machine with the following configura-

of landmarks for LLSVM and the budgeted size of support vectors for BSGD-SVM is determined by cross-validation method. If the result is from other literature, it will be followed by the citation.

Table 1: Details of Datasets

\begin{tabular}{l|l|l|l}
\hline Datasets & \# Instances & \# Dimensions & \# Classes \\
\hline REAL-SIM & 72,309 & 20,958 & 2 \\
\hline RCV1.BIN & 697,641 & 47,236 & 2 \\
\hline RCV1.MUL & 534,135 & 47,236 & 53 \\
\hline NEWS20.MUL & 19,928 & 62,061 & 20 \\
\hline URL0 & 20,000 & $3,231,955$ & 2 \\
\hline NEWS20.BIN & 19,996 & $1,355,191$ & 2 \\
\hline WEBSPAM & 350,000 & $16,609,143$ & 2 \\
\hline KDD2010 & $20,012,498$ & $29,890,095$ & 2 \\
\hline EPSILON & 500,000 & 2,000 & 2 \\
\hline AUT_AVN & 71,175 & 20,707 & 2 \\
\hline URL100 & $2,396,130$ & $3,231,961$ & 2 \\
\hline SECTOR & 9,619 & 55,197 & 105 \\
\hline
\end{tabular}

\footnotetext{
${ }^{3}$ http://www.tanmingkui.com/publications.html
} 
Table 2: Three different training/testing partitions

\begin{tabular}{l|l|l|l|c|c|c}
\hline \multirow{2}{*}{ Datasets } & \multicolumn{2}{|c|}{ Partition I } & \multicolumn{2}{c|}{ Partition II } & \multicolumn{2}{c}{ Partition III } \\
\cline { 2 - 7 } & \# Train set & \# Test set & \# Train set & \# Test set & \# Train set & \# Test set \\
\hline REAL-SIM & 32,309 & 40,000 & 57,847 & 14,462 & 65,078 & 7,231 \\
\hline RCV1.BIN & 677,399 & 20,242 & 558,110 & 139,531 & 627,880 & 69,761 \\
\hline RCV1.MUL & 518,571 & 15,564 & 427,308 & 106,827 & 480,720 & 53,415 \\
\hline NEWS20.MUL & 15,935 & 3,993 & 15,942 & 3,986 & 17,935 & 1,993 \\
\hline URL0 & 15,000 & 5,000 & 16,000 & 4,000 & 18,000 & 2,000 \\
\hline NEWS20.BIN & 9,996 & 10,000 & 15,997 & 3,999 & 17,996 & 2,000 \\
\hline WEBSPAM & 300,000 & 50,000 & 280,000 & 70,000 & 315,000 & 35,000 \\
\hline KDD2010 & $19,264,097$ & 748,401 & $16,010,000$ & $4,002,498$ & $17,338,000$ & $2,674,498$ \\
\hline EPSILON & 400,000 & 100,000 & 100,000 & 400,000 & 450,000 & 50,000 \\
\hline AUT_AVN & 40,000 & 31,175 & 56,940 & 14,235 & 64,058 & 7,117 \\
\hline URL100 & $1,976,130$ & 420,000 & $1,916,904$ & 479,226 & $2,156,517$ & 239,613 \\
\hline SECTOR & 6,412 & 3,207 & 7,695 & 1,924 & 8657 & 962 \\
\hline
\end{tabular}

\subsection{Performance Comparison of FSVD-H-ELM, SVD-H-ELM and Classical ELM}

For the classical ELM, SVD-H-ELM and the proposed FSVD-H-ELM algorithm, the Sigmoid activation function $g(x)=\frac{1}{1+e^{-\lambda x}}$ is considered, where $\lambda$ is set to ' 1 ' unless otherwise stated. The number of hidden nodes $L$ is then chosen by cross-validation method with step size 100, and only the obtained optimized number of hidden nodes are reported. For FSVD-H-ELM, as the subset size $n=200$ and $\rho=0.5$ usually can achieve the promising generalized performance at the lowest training time, we would set $n=200$ and $\rho=0.5$ unless otherwise sepecified. The details about the optimized parameter for settings for every datasets are reported in Table 3 .

In order to perform a comprehensive comparison, the experimental studies are carried out on three different training/testing partitions as shown in Table 2 . For each partition, 10 trials (in each trial the data will be shuffled before partition to attain different training and testing set) are conducted, and then, the experimental results including the average performance and standard derivation (mean \pm std.) of the proposed FSVD-HELM and state-of-the-art methods considered in the study are summarized in Tables 4 . 6 and 8 . To evaluate the performance difference of the considered algorithms, paired $t$-test [83] is introduced to obtain $t$ value and significant level $p$. The smaller the $p$ value 
Table 3: Parameters of FSVD-H-ELM, SVD-H-ELM and Classical ELM

\begin{tabular}{|c|c|c|c|c|c|c|c|c|c|c|c|c|}
\hline \multirow{3}{*}{ Datasets } & \multicolumn{6}{|c|}{ FSVD-H-ELM } & \multicolumn{3}{|c|}{ SVD-H-ELM } & \multicolumn{3}{|c|}{ Classical ELM } \\
\hline & \multicolumn{2}{|c|}{ P. I } & \multicolumn{2}{|c|}{ P. II } & \multicolumn{2}{|c|}{ P. III } & \multirow{2}{*}{$\begin{array}{l}\text { P. I } \\
L\end{array}$} & \multirow{2}{*}{$\begin{array}{l}\text { P. II } \\
L\end{array}$} & \multirow{2}{*}{$\begin{array}{l}\text { P. III } \\
L\end{array}$} & \multirow{2}{*}{$\begin{array}{l}\text { P. I } \\
L\end{array}$} & \multirow{2}{*}{$\begin{array}{l}\text { P. II } \\
L\end{array}$} & \multirow{2}{*}{$\begin{array}{l}\text { P. III } \\
L\end{array}$} \\
\hline & $L$ & $(n, \rho)$ & $L$ & $(n, \rho)$ & $L$ & $(n, \rho)$ & & & & & & \\
\hline REAL-SIM & 4000 & $(200,0.5)$ & 4000 & $(200,0.5)$ & 4000 & $(200,0.5)$ & 4000 & 4000 & 4000 & 5500 & 5500 & 5500 \\
\hline RCV1.BIN & 3000 & $(200,0.5)$ & 3000 & $(200,0.5)$ & 3000 & $(200,0.5)$ & 3000 & 3000 & 3000 & 4000 & 4000 & 4000 \\
\hline RCV1.MUL & 3000 & $(200,0.5)$ & 3000 & $(200,0.5)$ & 3000 & $(200,0.5)$ & 3000 & 3000 & 3000 & 4500 & 4500 & 4500 \\
\hline NEWS20.MUL & 1000 & $(200,0.5)$ & 1000 & $(200,0.5)$ & 1000 & $(200,0.5)$ & 1000 & 1000 & 1000 & 2000 & 2000 & 2000 \\
\hline URL0 & 600 & $(200,0.5)$ & 600 & $(200,0.5)$ & 600 & $(200,0.5)$ & 600 & 600 & 600 & 1000 & 1000 & 1000 \\
\hline NEWS20.BIN & 2000 & $(200,0.5)$ & 2000 & $(200,0.5)$ & 2000 & $(200,0.5)$ & 2000 & 2000 & 2000 & 2500 & 2500 & 2500 \\
\hline WEBSPAM & 1500 & $(200,0.5)$ & 1500 & $(200,0.5)$ & 1500 & $(200,0.5)$ & 1500 & 1500 & 1500 & 2500 & 2500 & 2500 \\
\hline KDD2010 & 150 & $(200,0.5)$ & 150 & $(200,0.5)$ & 150 & $(200,0.5)$ & 150 & 150 & 150 & 350 & 350 & 350 \\
\hline EPSILON & 600 & $(200,0.5)$ & 600 & $(200,0.5)$ & 600 & $(200,0.5)$ & 600 & 600 & 600 & 1500 & 1500 & 1500 \\
\hline AUT_AVN & 2000 & $(200,0.5)$ & 2000 & $(200,0.5)$ & 2000 & $(200,0.5)$ & 2000 & 2000 & 2000 & 3000 & 3000 & 3000 \\
\hline URL100 & 2000 & $(200,0.5)$ & 2000 & $(200,0.5)$ & 2000 & $(200,0.5)$ & 2000 & 2000 & 2000 & 3000 & 3000 & 3000 \\
\hline SECTOR & 2000 & $(200,0.5)$ & 2000 & $(200,0.5)$ & 2000 & $(200,0.5)$ & 2000 & 2000 & 2000 & 3000 & 3000 & 3000 \\
\hline
\end{tabular}

$L$ : the number of hidden nodes, $n$ : the subset size, $\rho$ : the selection ratio.

Table 4: Performance of FSVD-H-ELM, SVD-H-ELM and Classical ELM on Partition I

\begin{tabular}{|c|c|c|c|c|c|c|}
\hline \multirow{2}{*}{ Datasets } & \multicolumn{2}{|c|}{ FSVD-H-ELM } & \multicolumn{2}{|c|}{ SVD-H-ELM } & \multicolumn{2}{|c|}{ Classical ELM } \\
\hline & Training time (s) & Testing accuracy $(\%)$ & Training time (s) & Testing accuracy $(\%)$ & Training time (s) & Testing accuracy $(\%)$ \\
\hline REAL-SIM & 26 & $96.78 \pm 0.046$ & 153,413 & $\mathbf{9 6 . 8} \pm 0.044$ & 44 & $93.30 \pm 0.054$ \\
\hline RCV1.BIN & 1440 & $\mathbf{9 7 . 1 0} \pm 0.049$ & \multicolumn{2}{|c|}{$>48 \mathrm{~h}$} & 1102 & $94.40 \pm 0.053$ \\
\hline RCV1.MUL & 601 & $\mathbf{8 8 . 5 4} \pm 0.052$ & \multicolumn{2}{|c|}{$>48 \mathrm{~h}$} & 515 & $85.92 \pm 0.044$ \\
\hline NEWS20.MUL & 45 & $\mathbf{8 4 . 9 4} \pm 0.031$ & 132,245 & $82.93 \pm 0.024$ & 79 & $81.07 \pm 0.056$ \\
\hline URL0 & 240 & $\mathbf{9 7 . 1 8} \pm 0.042$ & 114,261 & $96.70 \pm 0.041$ & 213 & $95.43 \pm 0.048$ \\
\hline NEWS20.BIN & 414 & $95.80 \pm 0.050$ & 104,169 & $\mathbf{9 6 . 0 1} \pm 0.045$ & 400 & $87.85 \pm 0.062$ \\
\hline WEBSPAM & 8,154 & $\mathbf{9 8 . 8 3} \pm 0.034$ & \multicolumn{2}{|c|}{$O O M^{*}$} & 8,321 & $93.46 \pm 0.041$ \\
\hline KDD2010 & 1,769 & $\mathbf{8 8 . 7 4} \pm 0.047$ & \multicolumn{2}{|c|}{$O O M$} & 1,970 & $86.14 \pm 0.055$ \\
\hline EPSILON & 452 & $\mathbf{8 9 . 9 9} \pm 0.054$ & 34,678 & $89.31 \pm 0.047$ & 365 & $87.13 \pm 0.063$ \\
\hline AUT_AVN & 42 & $\mathbf{9 5 . 8 7} \pm 0.048$ & 14,822 & $95.48 \pm 0.042$ & 41 & $94.18 \pm 0.052$ \\
\hline URL100 & 610 & $\mathbf{9 9 . 0 1} \pm 0.043$ & \multicolumn{2}{|c|}{$>48 \mathrm{~h}$} & 589 & $97.73 \pm 0.051$ \\
\hline SECTOR & 19 & $\mathbf{9 3 . 9 5} \pm 0.054$ & 108,334 & $93.88 \pm 0.049$ & 11 & $91.13 \pm 0.063$ \\
\hline
\end{tabular}

* Out of memory.

Table 5: $t$ value and significant level $p$ of FSVD-H-ELM v.s. SVD-H-ELM and Classical ELM (Partition I)

\begin{tabular}{lll}
\hline & SVD-H-ELM (93.01\%) & Classical ELM (90.64\%) \\
\hline FSVD-H-ELM (93.89\%) & $t=0.3714, p>0.1$ & $t=1.6571,0.01>p>0.001$ \\
\hline
\end{tabular}


Table 6: Performance of FSVD-H-ELM, SVD-H-ELM and Classical ELM on Partition II

\begin{tabular}{|c|c|c|c|c|c|c|}
\hline \multirow{2}{*}{ Datasets } & \multicolumn{2}{|c|}{ FSVD-H-ELM } & \multicolumn{2}{|c|}{ SVD-H-ELM } & \multicolumn{2}{|c|}{ Classical ELM } \\
\hline & Training time (s) & Testing accuracy $(\%)$ & Training time (s) & Testing accuracy $(\%)$ & Training time (s) & Testing accuracy (\%) \\
\hline REAL-SIM & 46.4 & $97.59 \pm 0.045$ & \multicolumn{2}{|c|}{$>48 \mathrm{~h}$} & 79.2 & $93.86 \pm 0.053$ \\
\hline RCV1.BIN & 1187.4 & $\mathbf{9 7 . 0 4} \pm 0.046$ & \multicolumn{2}{|c|}{$>48 \mathrm{~h}$} & 937.2 & $93.83 \pm 0.054$ \\
\hline RCV1.MUL & 641 & $\mathbf{8 8 . 6 5} \pm 0.053$ & \multicolumn{2}{|c|}{$>48 \mathrm{~h}$} & 549.35 & $86.12 \pm 0.043$ \\
\hline NEWS20.MUL & 72 & $86.33 \pm 0.033$ & 211,592 & $84.18 \pm 0.022$ & 126.40 & $82.37 \pm 0.055$ \\
\hline URL0 & 256.0 & $\mathbf{9 7 . 4 2} \pm 0.040$ & 121,880 & $96.82 \pm 0.040$ & 227 & $95.87 \pm 0.046$ \\
\hline NEWS20.BIN & 662.4 & $96.16 \pm 0.051$ & 166670 & $96.21 \pm 0.051$ & 640 & $88.15 \pm 0.060$ \\
\hline WEBSPAM & 7583.2 & $\mathbf{9 8 . 8 1} \pm 0.032$ & \multicolumn{2}{|c|}{$O O M^{*}$} & 7738.5 & $93.16 \pm 0.042$ \\
\hline KDD2010 & 1470.2 & $\mathbf{8 8 . 7 2} \pm 0.047$ & \multicolumn{2}{|c|}{$O O M$} & 1637.3 & $86.11 \pm 0.053$ \\
\hline EPSILON & 113 & $\mathbf{8 9 . 1 2} \pm 0.051$ & 21,655 & $89.12 \pm 0.046$ & 91.25 & $86.87 \pm 0.063$ \\
\hline AUT_AVN & 59.17 & $\mathbf{9 5 . 9 8} \pm 0.045$ & 21099 & $95.63 \pm 0.043$ & 58.36 & $94.32 \pm 0.054$ \\
\hline URL100 & 581.1 & $\mathbf{8 9 . 9 7 \pm 0 . 0 4 4}$ & \multicolumn{2}{|c|}{$>48 \mathrm{~h}$} & 571.3 & $97.75 \pm 0.050$ \\
\hline SECTOR & 22.8 & $\mathbf{9 4 . 1 2} \pm 0.051$ & 118,653 & $93.96 \pm 0.051$ & 13.2 & $91.17 \pm 0.062$ \\
\hline
\end{tabular}

* Out of memory.

Table 7: $t$ value and significant level $p$ of FSVD-H-ELM v.s. SVD-H-ELM and Classical ELM (Partition II)

\begin{tabular}{lll}
\hline & SVD-H-ELM (92.65\%) & Classical ELM (90.80\%) \\
\hline FSVD-H-ELM (93.33\%) & $t=0.3622, p>0.1$ & $t=1.3487,0.01>p>0.001$ \\
\hline
\end{tabular}

Table 8: Performance of FSVD-H-ELM, SVD-H-ELM and Classical ELM on Partition III

\begin{tabular}{|c|c|c|c|c|c|c|}
\hline \multirow{2}{*}{ Datasets } & \multicolumn{2}{|c|}{ FSVD-H-ELM } & \multicolumn{2}{|c|}{ SVD-H-ELM } & \multicolumn{2}{|c|}{ Classical ELM } \\
\hline & Training time (s) & Testing accuracy $(\%)$ & Training time (s) & Testing accuracy (\%) & Training time (s) & Testing accuracy (\%) \\
\hline REAL-SIM & 26 & $97.98 \pm 0.045$ & \multicolumn{2}{|c|}{$>48 \mathrm{~h}$} & 90.4 & $94.21 \pm 0.052$ \\
\hline RCV1.BIN & 1076.1 & $\mathbf{9 7 . 0 8} \pm 0.048$ & \multicolumn{2}{|c|}{$>48 \mathrm{~h}$} & 1022.3 & $94.16 \pm 0.055$ \\
\hline RCV1.MUL & 721.2 & $\mathbf{8 8 . 5 4} \pm 0.053$ & \multicolumn{2}{|c|}{$>48 \mathrm{~h}$} & 618 & $85.92 \pm 0.043$ \\
\hline NEWS20.MUL & 81 & $\mathbf{8 4 . 9 4} \pm 0.029$ & 132,245 & $82.93 \pm 0.023$ & 142.2 & $81.07 \pm 0.055$ \\
\hline URL0 & 288 & $\mathbf{9 7 . 1 8} \pm 0.043$ & 114,261 & $96.70 \pm 0.040$ & 255.6 & $95.43 \pm 0.047$ \\
\hline NEWS20.BIN & 745.2 & $95.80 \pm 0.050$ & 187500 & $\mathbf{9 6 . 0 1} \pm 0.046$ & 70 & $87.85 \pm 0.063$ \\
\hline WEBSPAM & 8561.7 & $\mathbf{9 8 . 8 3} \pm 0.032$ & \multicolumn{2}{|c|}{$O O M^{*}$} & 8737.1 & $93.46 \pm 0.040$ \\
\hline KDD2010 & 1532.6 & $\mathbf{8 8 . 7 4} \pm 0.049$ & \multicolumn{2}{|c|}{$O O M$} & 1706.8 & $86.14 \pm 0.057$ \\
\hline EPSILON & 508.5 & $\mathbf{8 9 . 9 9} \pm 0.052$ & 39013 & $89.31 \pm 0.049$ & 410.625 & $87.13 \pm 0.064$ \\
\hline AUT_AVN & 67.26 & $95.87 \pm 0.049$ & 23737 & $95.48 \pm 0.043$ & 65.6 & $94.18 \pm 0.053$ \\
\hline URL100 & 663 & $\mathbf{9 9 . 0 3} \pm 0.042$ & \multicolumn{2}{|c|}{$>48 \mathrm{~h}$} & 642.7 & $97.73 \pm 0.052$ \\
\hline SECTOR & 25.6 & $\mathbf{9 4 . 2 5} \pm 0.055$ & 119,312 & $93.98 \pm 0.047$ & 14.85 & $91.19 \pm 0.062$ \\
\hline
\end{tabular}

*Out of memory.

Table 9: $t$ value and significant level $p$ of FSVD-H-ELM v.s. SVD-H-ELM and Classical ELM (Partition III)

\begin{tabular}{lll}
\hline & SVD-H-ELM $(92.4 \%)$ & Classical ELM $(90.7 \%)$ \\
\hline FSVD-H-ELM $(94.02 \%)$ & $t=0.4112, p>0.1$ & $t=1.7223,0.01>p>0.001$ \\
\hline
\end{tabular}


the more significant the difference. $t$ value can be computed as follows:

$$
t=\frac{\overline{\boldsymbol{a}}_{i}-\overline{\boldsymbol{a}}_{j}}{\sqrt{\frac{v_{i}^{2}}{n_{i}}+\frac{v_{j}^{2}}{n_{j}}}}
$$

where $\boldsymbol{a}_{i}=a_{i, 1}, a_{i, 2}, \ldots, a_{i, 12}$ denote the testing accuracies on the twelve datasets of $i$-th algorithm, $\overline{\boldsymbol{a}}_{i}$ and $\overline{\boldsymbol{a}}_{j}$ denotes the mean value of $\boldsymbol{a}_{i}$ and $\boldsymbol{a}_{j}, v_{i}^{2}$ and $v_{j}^{2}$ represent the variance of $\boldsymbol{a}_{i}$ and $\boldsymbol{a}_{j}$, and $n_{i}$ and $n_{j}$ denotes the number of datasets (here $n_{i}=$ $n_{j}=12$ ). Then, by checking $t$-table [83], the significant level $p$ is obtained by $t$ value. The obtained $t$-test results are summarized in Tables 5, 7 and 9. With respect to the classical ELM, the training time incurred by FSVD-H-ELM and the classical ELM are observed to be competitive to one another on the twelve data sets. This may be attributed to the fact that, although the SVD operation of FSVD-H-ELM is originally thought to be more computationally intensive than the random assignment of hidden nodes in the classical ELM, it turns out that as the classical ELM often needs many more hidden nodes than is required by the FSVD-H-ELM, the overall computation time incurred by the classical ELM is equivalently high due to the need to perform the Moore-Penrose generalized inverse on a larger matrix (in the latter) when deriving the output weights. From Tables 5, 7 and 9, we can find that, on three partitions, the average testing accuracy, $t$-value and significant level $p$ of FSVD-H-ELM and classical ELM are $93.89 \%$ vs. $90.64 \%(t=1.6571,0.01>p>0.001), 93.33 \%$ vs. $90.80 \%(t$ $=1.3487,0.01>p>0.001), 94.02 \%$ vs. $90.7 \%(t=1.7223$ and $0.01>p>0.001)$ respectively. This indicates that FSVD-H-ELM showcased significant improvements in the test performance with respect to classical ELM. FSVD-H-ELM outperformed classical ELM with 2 8\% improvements. For example, on the Big dimensional dataset NEWS 20.BIN with Partition I, FSVD-H-ELM achieved a test performance of $95.80 \%$, which is a significant improvement over the classical ELM accuracy of $87.85 \%$. On the Small dimensional datasets REAL-SIM and RCV1.BIN, FSVD-H-ELM achieved test accuarcy performances of $96.78 \%$ and $97.1 \%$, respectively, which are also superior to that of the classical ELM at $93.3 \%$ and $94.4 \%$, respectively. These indicate that the FSVD-H-ELM is able to appropriately capture the structural characteristic of the data much better than the classical ELM (which uses random hidden nodes). 
ly obtained on seven of the small instance datasets REAL-S IM, NEWS20.MUL, URLO, NEWS 20.BIN and AUT_AVN. On the Big instance datasets, RCV1.BIN, URL100 and RCV1.MULT, the simulations failed to complete despite 2 days of computations (48 hours). On WEBSPAM and KDD2010, for instance, SVD-H-ELM went into the state of "out of memory (OOM)". From these results, it is clear that SVD-H-ELM is incapable of handling the Big instance dataset well as expected, thus the need for our introduction of the divide-and-conquer approximation scheme to arrive at the efficient FSVDH-ELM. The results obtained indicate that FSVD-H-ELM is thousands of times more efficient than the SVD-H-ELM counterpart in most cases. For example, on REAL-SIM, SVD-H-ELM consumed a CPU wall clock time of 251,212 seconds to model the data, while FSVD-H-ELM only took 26 seconds to perform the same task. More importantly, the higher efficiency of FSVD-H-ELM is attained at no form of degradation in the test accuracy performance over SVD-H-ELM, in spite of the approximation. Interestingly, FSVD-H-ELM exhibits excellent test accuracy performance in most cases. As shown in Tables 5, 7 and 9, the average testing accuracy of FSVD-H-ELM vs. SVDH-ELM on three partitions are $93.89 \%$ vs. $93.01 \%(t=0.3714, p>0.1), 93.33 \%$ vs. $92.65 \%(t=0.3622, p>0.1)$, and $94.02 \%$ vs. $92.4 \%(t=0.4112$ and $p>0.1)$ respectively. These showcased the improved test accuracies over the SVD-H-ELM. For example, on the URLO dataset with Partition I, FSVD-H-ELM achieved a test accuracy of $97.18 \%$, which is slightly superior to the SVD-H-ELM of $96.70 \%$. These results thus highlights the high efficacy of the FSVD-H-ELM in handling large-scale datasets. It is worth noting that the reasons for the reported high performances of the FSVD-H-ELM may be attributed to the ensemble like structure of FSVD-H-ELM, which integrates multiple SVD de-noised data subsets. Both ensemble and de-noising method have been proven to be effective for improving the resultant test accuracy per-

With respect to the SVD-H-ELM, experimental results have only been successfulformance in the literature [78]. FSVD-H-ELM thus inherits their benefits to generate the reported stable and excellent performances. Moreover, FSVD-H-ELM also eliminates the 'out of memory' problem that exists when dealing with Big instance \& Big dimensional dataset such as WEBSPAM and KDD2010 and shown to work with them efficiently and elegantly. It also showcased a test accuracy of $88.74 \%$ on the KDD 2010 
dataset that is not only superior to all the other state-of-the-art algorithms considered, it also completed the task within a short time span of 1,769 seconds training time.

\subsection{Performance Comparison of FSVD-H-ELM to the Other State-of-the-art Machine}

\section{Learning Methods}

In this subsection, a comprehensive performance comparison of the FSVD-H-ELM is conducted against other state-of-the-art large-scale machine learning algorithms including the FGM[81], LLSVM, BSGD[56] and others. The performance results in terms of training time and testing accuracy are then summarized in Table 10 . Here we reference and report the results from the relevant literatures if available. From the results, the following observations have been made:

1. With respect to the FGM, which is a recently proposed state-of-the-art algorithm for solving Big dimensional dataset, FSVD-H-ELM exhibited competitive generalization performances. Notably, although FGM is implemented in C++, FSVD-H-ELM is shown to incur shorter training time costs than FGM in many cases, despite the latter operating under a MATLAB environment. For example on NEWS2 0.BIN, FSVD-H-ELM took 414 seconds to achieve a test accuracy of 95.80\%, while FGM took 454 seconds to achieve a similar accuracy of $95.08 \%$. Furthermore, FGM is specially designed for two-class problems and cannot support multi-class problems directly, unless some extension is made. A possible solution is to first decompose the multi-class problem into multiple binary-class problems, train the classifiers to solve these problems, and then reconstruct the solution of the multi-class problem from the outputs attained by the classifiers. In contrast, FSVD-H-ELM can be convenient used for binary-class, multi-class and regression problems seamlessly.

2. With respect to the other state-of-the-art algorithms, FSVD-H-ELM has been observed to attain better generalization performances than TSVM [84], LLSVM[57], BSGD[56], DTSVM[89] and AMM[55] on REAL-S IM, RCV1.MUL, NEWS2 0.MUL, NEWS20.BIN and KDD2010 at much lower training time. Although on the RCV1.BIN, URLO and WEBSPAM datasets, FSVD-H-ELM did not perform significantly better than SVM (RBF), FGM and DTSVM, however, its test accu- 
Table 10: Performance evaluation of FSVD-H-ELM and other state-of-the-art machine learning algorithms

\begin{tabular}{|c|c|c|c|}
\hline Datasets & Methods & Trainingtime (s) & Testingaccuracy (\%) \\
\hline \multicolumn{4}{|c|}{ Big instance \& Small dimension } \\
\hline \multirow{4}{*}{ REAL-SIM } & FSVD-H-ELM & 26 & 96.78 \\
\hline & FGM & 675 & 96.52 \\
\hline & TSVM 84 & 373 & 93.10 \\
\hline & $\mathrm{DA} 84$ & 1,129 & 92.80 \\
\hline \multirow{5}{*}{ RCV1.BIN } & FSVD-H-ELM & 1440 & 97.10 \\
\hline & FGM & 10,923 & 97.42 \\
\hline & LLSVM 85 & 1,800 & 95.77 \\
\hline & BSGD-SVM 56 & 5,400 & 97.08 \\
\hline & LibSVM (RBF) 86 & 72,720 & 97.83 \\
\hline \multirow{6}{*}{ EPSILON } & FSVD-H-ELM & 452 & 89.89 \\
\hline & $\mathrm{CDN} 87$ & 3000 & 89.82 \\
\hline & newGLMNET 87 & 500 & 89.82 \\
\hline & FGM & 1,912 & 89.64 \\
\hline & LLSVM & 3,870 & 88.71 \\
\hline & BSGD-SVM(5 pass) & 6,896 & 89.58 \\
\hline \multirow{6}{*}{ URL100 } & FSVD-H-ELM & 640 & 98.97 \\
\hline & AMM(Batch) & 1214 & 98.13 \\
\hline & AMM(Online) & 1465 & 98.10 \\
\hline & & 1,213 & 98.22 \\
\hline & LLSVM & 2,512 & 98.17 \\
\hline & BSGD-SVM & 1,701 & 98.32 \\
\hline \multicolumn{4}{|c|}{ Small instance \& Small dimension } \\
\hline \multirow{5}{*}{ RCV1.MUL } & FSVD-H-ELM & 610 & 88.54 \\
\hline & K-Pegasos 88 & N.A. & 84.50 \\
\hline & K-binaryLR 88 & N.A. & 83.00 \\
\hline & Multi-class LR 88 & N.A. & 88.50 \\
\hline & FGM & \multicolumn{2}{|l|}{$\begin{array}{l}\text { Unsupported } \\
\text { * } \\
\end{array}$} \\
\hline \multirow{6}{*}{ NEWS20.MUL } & FSVD-H-ELM & 45 & 84.94 \\
\hline & LASVM 89 & 23,339 & 83.10 \\
\hline & DTSVM 89 & 3,053 & 83.22 \\
\hline & $\mathrm{CBD} 89$ & 39,590 & 75.23 \\
\hline & Bagging 89 & 35,176 & 76.55 \\
\hline & FGM & \multicolumn{2}{|l|}{ Unsupported $\stackrel{*}{*}$} \\
\hline \multirow{7}{*}{ AUT_AVN } & FSVD-H-ELM & 42 & 95.87 \\
\hline & FGM & 218 & 95.72 \\
\hline & AMM(Batch) & 321 & 94.23 \\
\hline & AMM(Online) & 346 & 94.10 \\
\hline & FGM & 206 & 95.22 \\
\hline & LLSVM & 531 & 94.97 \\
\hline & BSGD-SVM & 318 & 95.32 \\
\hline \multicolumn{4}{|c|}{ Small instance \& Big dimension } \\
\hline \multirow{4}{*}{ NEWS20.BIN } & FSVD-H-ELM & 414 & 95.80 \\
\hline & FGM & 454 & 95.08 \\
\hline & Forgetron $[90]$ & N.A. & 90.00 \\
\hline & Projectron++ 90 & N.A. & 95.00 \\
\hline \multirow{3}{*}{ URLO } & FSVD-H-ELM & 240 & 97.18 \\
\hline & FGM & 727 & 97.38 \\
\hline & AMM & 224 & 97.13 \\
\hline \multicolumn{4}{|c|}{ Big instance \& Big dimension } \\
\hline & FSVD-H-ELM & 8154 & 98.83 \\
\hline WEBSPAM & CART 89] & 29,332 & 98.44 \\
\hline 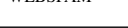 & DTSVM 89 & 63,015 & 99.03 \\
\hline & FSVD-H-ELM & 1,769 & 88.74 \\
\hline & L1-SVM 81] & 1,500 & 88.40 \\
\hline KDD2010 & FGM 81 & 1,870 & 88.70 \\
\hline & PROX-SLR 81$]$ & 1,000 & 88.40 \\
\hline & FSVD-H-ELM & 19 & 93.95 \\
\hline & LibSVM(OVA) 91 & - & 92.80 \\
\hline SECTOR & $\mathrm{NB}(\mathrm{OVA})$ 91] & - & 64.30 \\
\hline & LibSVM(BCH 63$) 91$ & - & 93.3 \\
\hline & $\mathrm{NB}(\mathrm{BCH} 63) 91$ & - & 87.2 \\
\hline & FGM & Unsupported $*$ & \\
\hline
\end{tabular}

* FGM is especially designed for two-class problems and can not be applicable for multi-class problems directly. It needs some extending methods such as decomposing the multi-class problem to several two-class problems. 
racies of $97.10 \%$ on RCV1.BIN, $97.18 \%$ on URLO and $98.83 \%$ on WEBSPAM, are competitive to the optimal results of the state-of-the-art methods, which are $97.83 \%, 97.38 \%$ and $99.03 \%$, respectively. More important, FSVD-H-ELM is able to attain the improved or competitive test accuracies at a much shorter training time than the state-of-the-art algorithms considered in most cases. In particular, on the Big instance \& Big dimensional dataset KDD2010, which has more than 29 million features and 190 millon training instance samples, FSVDH-ELM is very competitive in terms of test accuracy. It showcased a testing accuracy of $88.74 \%$, which is an improvement over the L1-SVM (88.4\%), FGM (88.7\%) and PROX-SLR (88.4\%).

\subsection{Stability of The Divide \& Conquer Fast Approximation}

In this subsection, the stability of the divide \& conquer fast approximation scheme proposed is studied and visualized. For each dataset, 20 independent experimental trials have been conducted and the performance accuracies are summarized in Fig. 4 In all the trials, The parameter settings (including subset size and selection ratio) is set as in Table 3 The data subsets are constructed via random sampling from the original dataset and the SVD hidden nodes are derived subsequently. The results in Fig 4 demonstrate excellent robustness of the proposed FSVD-H-ELM, where the testing accuracies are observed to remains stable across all 20 independent experimental trails.

\subsection{The Effects of Data Subset Size n on SVD-H-ELM Performance}

Here we conduct further experimental study to evaluate the influence of data subset size $n$ on FSVD-H-ELM performances, i.e., testing accuracy and computational time. Fig 5 summarizes the testing accuracy and training time performances of the FSVDH-ELM for different data subset size $n=[5,10,50,200,700,1100,1500,3000]$ on the six data sets. From the figures, it is worth noting that the testing accuracy can be observed to remain relatively stable for different data subset size $n$, which highlights the robustness of the proposed FSVD-H-ELM. The training time, however, varies significantly for different subset size $n$. In particular, when $n$ gets too large or too small, 

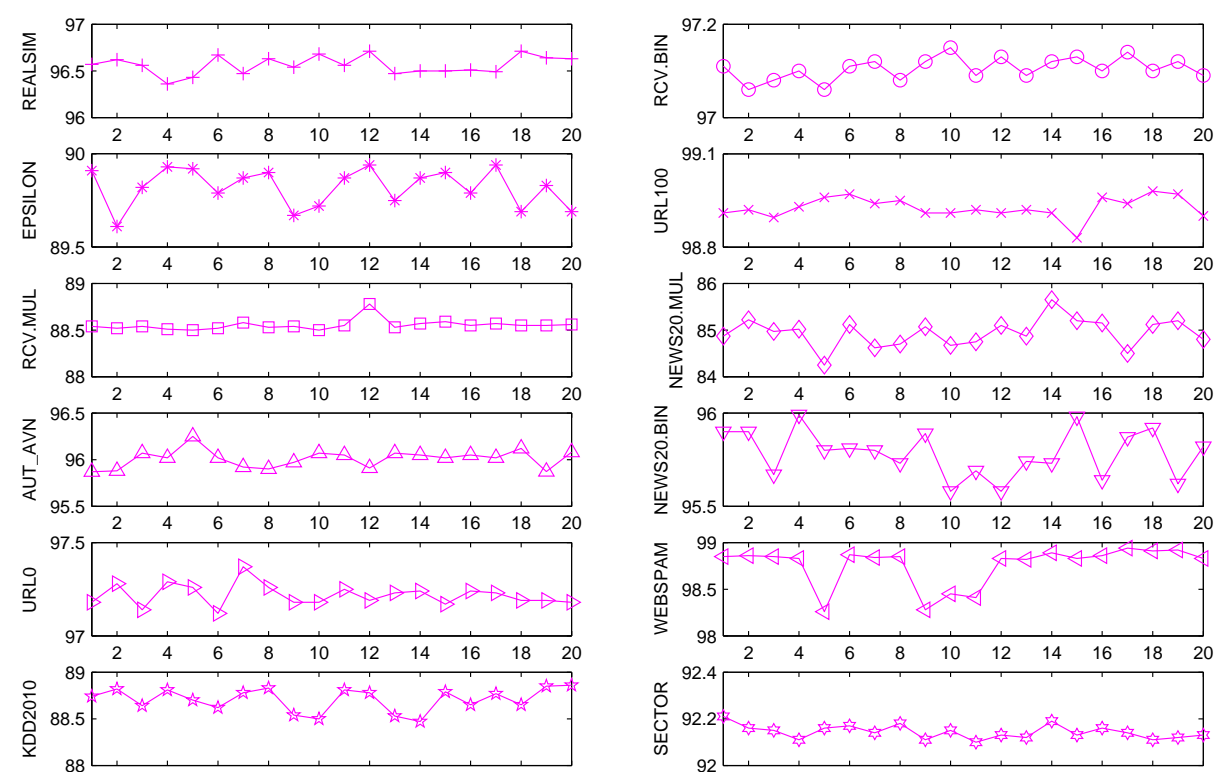

Figure 4: FSVD-H-ELM testing accuracies of 20 trials on 12 data sets. In every trials the subsets are different. The testing accuracies are observed to remain stable across all 20 independent experimental trails. 


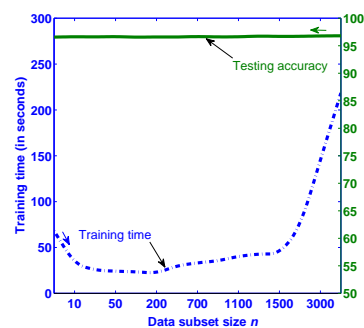

(a) REAL-SIM

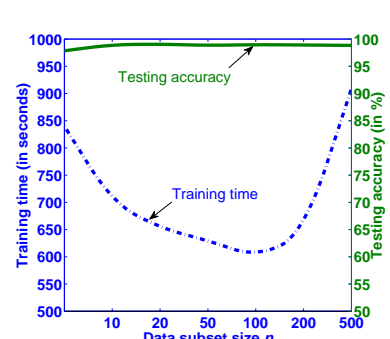

(d) URL100

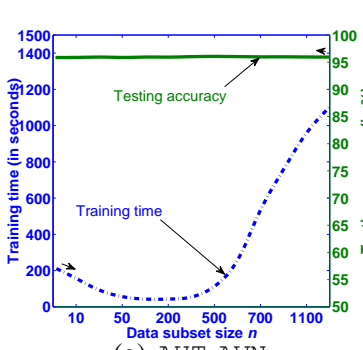

(g) AUT_AVN

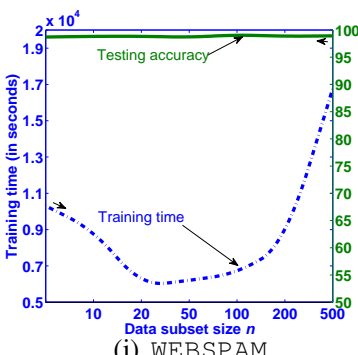

(j) WEBSPAM

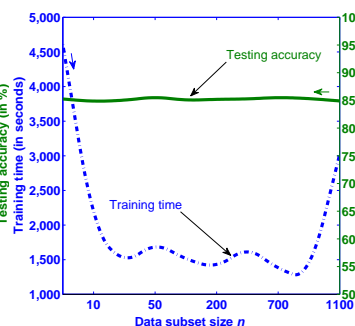

(b) RCV1.BIN

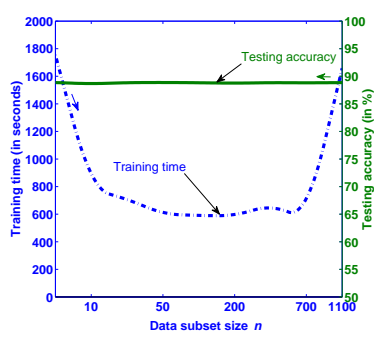

(e) RCV1.MUL

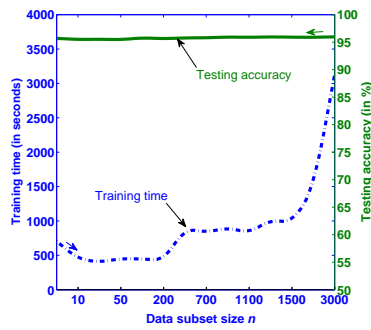

(h) NEWS20.BIN

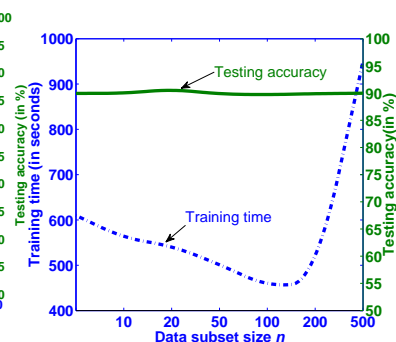

(c) EPSLION

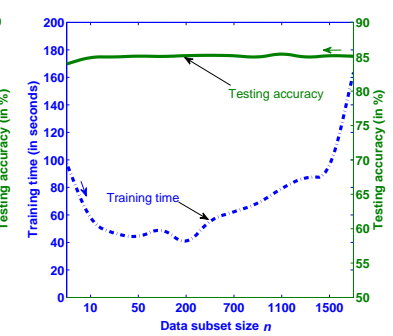

(f) NEWS20.MUL

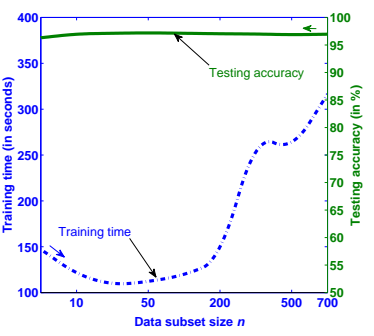

(i) URLO

Figure 5: Training time(in seconds) and Testing accuracy (in \%) w.r.t different data subset size $n$. In particular, the testing accuracy can be observed to remain relatively stable for different data subset sizes $n$. The training time, however, varies significantly with $n$. 
the computational time is noted to greatly increase. This can be comprehended with a complexity analysis of the FSVD-H-ELM.

Considering a data subset of size $n$, the original dataset of $N$ data samples is first divided into $\left\lfloor\frac{N}{n}\right\rfloor$ data subsets $\left\{\mathbf{X}_{r}\right\}_{r=1}^{\left\lfloor\frac{N}{n}\right\rfloor}$. Assuming $l$ hidden nodes are generated by each data subset of size $n$. A concatenation of the hidden nodes derived from each of the $s=\left\lceil\frac{L}{l}\right\rceil$ randomly chosen data subsets in $\left\{\mathbf{X}_{r}\right\}_{r=1}^{\left\lfloor\frac{N}{n}\right\rfloor}$ then forms a trained FSVD-

H-ELM network of $L$ hidden nodes. Note that the time complexity of SVD on a data subset of size $n$ and dimension $d$ is $\mathcal{O}\left(n^{2} d\right)$, while other operations such as data subset retrieval takes a constant cost of $\Theta$. Thus, FSVD-H-ELM has overall time complexity of $\mathcal{O}\left(\frac{L}{l} \cdot\left(n^{2} d+\Theta\right)\right) \approx \mathcal{O}\left(L d \cdot n+L \Theta \cdot \frac{1}{n}\right)$. From the analysis, it is clear that when $n$ gets too large, the first term of the complexity, i.e., the cost of SVD on $s$ data subsets dominates. Conversely, when $n$ gets too small, the second term, i.e., the data subset retrieval time dominates in comparison. As a result, the total computational cost of FSVD-H-ELM at $\mathcal{O}\left(L d \cdot n+L \Theta \cdot \frac{1}{n}\right)$ inflates when $n$ is either too large or too small. In this manner, an appropriate data subset size that accords to the computational requirements of the user should be chosen. Based on our experimental study, a data subset size in the range of $n \in[50,500]$ is noted to function well in general.

\section{Conclusion}

This paper has proposed the FSVD-H-ELM, which is designed to work with Big dimensional data. In contrast to existing approaches, we have embedded singular value decomposition hidden nodes into the classical ELM, while preserving the benefits of ELM where hidden nodes need not be tuned. In our study, SVD hidden nodes have been shown to be capable of capturing the structural properties of Big dimensional data, thus providing a more meaningful representation in the hidden layer of ELM. To address the high computational complexity of SVD, especially when dealing with large-scale problems, i.e., Big instance and Big dimensional data, a divide and conquer fast approximation scheme is made available in FSVD-H-ELM. The SVD hidden nodes which are derived from multiple random data subsets are used in place of the SVD hidden nodes derived from the entire data set, thus making it highly s- 
calable to Big data. Comprehensive experiments have been conducted to validate the proposed FSVD-H-ELM on benchmark data sets. Further comparisons against several other state-of-the-art approaches verified and confirmed the superior generalization performance, robustness and efficiency of the proposed FSVD-H-ELM for solving Big dimensional data. Here we use SVD to obtain the hidden nodes, however, besides the use of SVD, it is worth noting that one may also choose other feature learning schemes including Non-negative Matrix Factorization (NMF), Kernel Principle Analysis (KPCA) and so on according to the problem of interest and requirement. This thus forms the immediate interest of our future works on Big Data analytics.

\section{Acknowledgment}

This work is partially supported by the ASTAR Thematic Strategic Research Programme (TSRP) Grant No. 1121720013, the Computational Intelligence Research Laboratory at NTU and conducted in collaboration with the Innovation fund research group 61221063; National Science Foundation of China 61100166, 61202184, 91118005, 91218301; Shaanxi New Star of Science \& Technology 2013KJXX-29; New Star Team of Xian University of Posts \& Telecommunications; Provincial Key Disciplines Construction Fund of General Institutions of Higher Education in Shaanxi.

\section{References}

[1] Y.-T. Zhai, Y.-S. Ong, I. W. Tsang, The emerging big dimensionality, IEEE Computational Intelligence Magazine 9 (3) (2014) 14-26.

[2] R. Sarunas, P. Vitalijus, On dimensionality, sample size, classification error, and complexity of classification algorithm in pattern recognition, IEEE Transactions on Pattern Analysis and Machine Intelligence 2 (3) (1980) 242-252.

[3] P. Hanchuan, L. Fulmi, D. Chris, Feature selection based on mutual information criteria of max-dependency, max-relevance, and min-redundancy, IEEE Transactions on Pattern Analysis and Machine Intelligence 27 (8) (2005) 1226-1238. 
q [4] I. Chaturvedi, Y.-S. Ong, R. V. Arumugam, Deep transfer learning for classification of time-delayed gaussian networks Signal Processing 110 (2015) 250 - 262, machine learning and signal processing for human pose recovery and behavior analysis. doi:http://dx.doi.org/10.1016/j.sigpro.2014.09. 009

URL http://www.sciencedirect.com/science/article/pii/ S0165168414004198

[5] C.-W. Seah, I. W. Tsang, Y.-S. Ong, Transfer ordinal label learning, Neural Networks and Learning Systems, IEEE Transactions on 24 (11) (2013) 1863-1876.

[6] L. Feng, Y. Ong, M. Lim, I. Tsang, Memetic search with inter-domain learning: A realization between cvrp and carp, Evolutionary Computation, IEEE Transactions on PP (99) (2014) 1-1. doi:10.1109/TEVC.2014.2362558.

[7] C.-W. Seah, Y.-S. Ong, I. Tsang, Combating negative transfer from predictive distribution differences, Cybernetics, IEEE Transactions on 43 (4) (2013) 11531165. doi:10.1109/TSMCB.2012.2225102

[8] C.-W. Seah, I. W. Tsang, Y.-S. Ong, Transductive ordinal regression, Neural Networks and Learning Systems, IEEE Transactions on 23 (7) (2012) 1074-1086.

[9] G. Schaefer, B. Krawczyk, M. E. Celebi, H. Iyatomi, An ensemble classification approach for melanoma diagnosis, Memetic Computing 6 (4) (2014) 233-240.

[10] J. A. Bullinaria, K. AlYahya, Artificial bee colony training of neural networks: comparison with back-propagation, Memetic Computing 6 (3) (2014) 171-182.

[11] D. Rummelhart, Learning representations by back-propagation errors, Nature 323 (1986) 533-536.

[12] M. A. Salama, A. E. Hassanien, K. Revett, Employment of neural network and rough set in meta-learning, Memetic Computing 5 (3) (2013) 165-177.

[13] G.-B. Huang, Q.-Y. Zhu, C.-K. Siew, Extreme learning machine: theory and applications, Neurocomputing 70 (1) (2006) 489-501. 
[14] G.-B. Huang, Z. Bai, L. L. C. Kasun, C. M. Vong, Local receptive fields based extreme learning machine, Computational Intelligence Magazine, IEEE 10 (2) (2015) 18-29.

[15] L. L. C. Kasun, H. Zhou, G.-B. Huang, C. M. Vong, Representational learning with extreme learning machine for big data, IEEE Intelligent Systems 28 (6) (2013) 1-4.

[16] G.-B. Huang, L. Chen, C.-K. Siew, Universal approximation using incremental constructive feedforward networks with random hidden nodes, IEEE Transactions on Neural Networks 17 (4) (2006) 879-892.

[17] G.-B. Huang, H. Zhou, X. Ding, R. Zhang, Extreme learning machine for regression and multiclass classification, IEEE Transactions on Systems, Man, and Cybernetics, Part B: Cybernetics 42 (2) (2012) 513-529.

[18] H. Chen, J. Peng, Y. Zhou, L. Li, Z. Pan, Extreme learning machine for ranking: Generalization analysis and applications, Neural Networks 53 (0) (2014) 119 126.

[19] A. Bueno-Crespo, P. J. Garca-Laencina, J.-L. Sancho-Gmez, Neural architecture design based on extreme learning machine, Neural Networks 48 (0) (2013) 19 24.

[20] M. Fernandez-Delgado, E. Cernadas, S. Barro, J. Ribeiro, J. Neves, Direct kernel perceptron (dkp): Ultra-fast kernel elm-based classification with non-iterative closed-form weight calculation, Neural Networks 50 (0) (2014) 60 - 71.

[21] G.-B. Huang, X. Ding, H. Zhou, Optimization method based extreme learning machine for classification, Neurocomputing 74 (1) (2010) 155-163.

[22] H.-J. Rong, Y.-S. Ong, A.-H. Tan, Z. Zhu, A fast pruned-extreme learning machine for classification problem, Neurocomputing 72 (13) (2008) 359 - 366, machine Learning for Signal Processing (MLSP 2006) / Life System Modelling, Simulation, and Bio-inspired Computing (LSMS 2007). 
doi:http://dx.doi.org/10.1016/j.neucom.2008.01.005.

URL http://www.sciencedirect.com/science/article/pii/

[23] E. Cambria, G.-B. Huang, L. L. C. Kasun, H. Zhou, C. M. Vong, J. Lin, J. Yin, Z. Cai, Q. Liu, K. Li, V. C. Leung, L. Feng, Y.-S. Ong, M.-H. Lim, A. Akusok, A. Lendasse, F. Corona, R. Nian, Y. Miche, P. Gastaldo, R. Zunino, S. Decherchi, X. Yang, K. Mao, B.-S. Oh, J. Jeon, K.-A. Toh, A. B. J. Teoh, J. Kim, H. Yu, Y. Chen, J. Liu, Extreme learning machines [trends controversies], Intelligent Systems, IEEE 28 (6) (2013) 30-59. doi:10.1109/MIS . 2013 . 140

[24] E. Kan, M. Lim, Y. Ong, A. Tan, S. Yeo, Extreme learning machine terrainbased navigation for unmanned aerial vehicles, Neural Computing and Applications 22 (3-4) (2013) 469-477. doi:10.1007/s00521-012-0866-9. URL http://dx.doi.org/10.1007/s00521-012-0866-9

[25] W.-Y. Deng, Q.-H. Zheng, Z.-M. Wang, Cross-person activity recognition using reduced kernel extreme learning machine, Neural Networks 53 (0) (2014) 1 - 7.

[26] J. Butcher, D. Verstraeten, B. Schrauwen, C. Day, P. Haycock, Reservoir computing and extreme learning machines for non-linear time-series data analysis, Neural Networks 38 (0) (2013) $76-89$.

[27] Y. Xu, Z. Y. Dong, J. H. Zhao, P. Zhang, K. P. Wong, A reliable intelligent system for real-time dynamic security assessment of power systems, Power Systems, IEEE Transactions on 27 (3) (2012) 1253-1263.

[28] B. P. Chacko, V. V. Krishnan, G. Raju, P. B. Anto, Handwritten character recognition using wavelet energy and extreme learning machine, International Journal of Machine Learning and Cybernetics 3 (2) (2012) 149-161.

[29] A. Mohammed, R. Minhas, Q. J. Wu, M. Sid-Ahmed, Human face recognition based on multidimensional pca and extreme learning machine, Pattern Recognition 44 (10) (2011) $2588-2597$. 
[30] S. Saraswathi, S. Sundaram, N. Sundararajan, M. Zimmermann, M. NilsenHamilton, Icga-pso-elm approach for accurate multiclass cancer classification resulting in reduced gene sets in which genes encoding secreted proteins are highly represented, IEEE/ACM Transactions on Computational Biology and Bioinformatics 8 (2) (2011) 452-463.

[31] J. Luo, C.-M. Vong, P.-K. Wong, Sparse bayesian extreme learning machine for multi-classification, Neural Networks and Learning Systems, IEEE Transactions on 25 (4) (2014) 836-843.

[32] X. Liu, S. Lin, J. Fang, Z. Xu, Is extreme learning machine feasible? a theoretical assessment (part i), Neural Networks and Learning Systems, IEEE Transactions on 26 (1) (2015) 7-20.

[33] S. Lin, X. Liu, J. Fang, Z. Xu, Is extreme learning machine feasible? a theoretical assessment (part ii), Neural Networks and Learning Systems, IEEE Transactions on 26 (1) (2015) 21-34.

[34] H. Jaeger, The echo state approach to analysing and training recurrent neural networks-with an erratum note, Bonn, Germany: German National Research Center for Information Technology GMD Technical Report 148 (2001) 1-47.

[35] H. Jaeger, H. Haas, Harnessing nonlinearity: Predicting chaotic systems and saving energy in wireless communication, Science 304 (5667) (2004) 78-80.

[36] W. Maass, T. Natschläger, H. Markram, Real-time computing without stable states: A new framework for neural computation based on perturbations, Neural computation 14 (11) (2002) 2531-2560.

[37] D. Lowe, Adaptive radial basis function nonlinearities, and the problem of generalisation, in: Artificial Neural Networks, 1989., First IEE International Conference on (Conf. Publ. No. 313), 1989, pp. 171-175.

[38] W. Schmidt, M. Kraaijveld, R. Duin, Feedforward neural networks with random weights, in: Pattern Recognition, 1992. Vol.II. Conference B: Pattern Recognition 
Methodology and Systems, Proceedings., 11th IAPR International Conference on, 1992, pp. 1-4.

[39] Y. Pao, Adaptive pattern recognition and neural networks, Reading, MA (US); Addison-Wesley Publishing Co., Inc., 1989.

[40] G.-B. Huang, What are extreme learning machines? filling the gap between frank rosenblatt's dream and john von neumann's puzzle, Cognitive Computation in press.

[41] G. Huang, G.-B. Huang, S. Song, K. You, Trends in extreme learning machines: A review, Neural Networks 61 (0) (2015) $32-48$.

[42] G.-B. Huang, An insight into extreme learning machines: Random neurons, random features and kernels, Cognitive Computation 6 (3) (2014) 376-390.

[43] B. Widrow, A. Greenblatt, Y. Kim, D. Park, The no-prop algorithm: A new learning algorithm for multilayer neural networks, Neural Networks 37 (2013) 182188.

[44] Z. Bai, G.-B. Huang, D. Wang, H. Wang, M. B. Westover, Sparse extreme learning machine for classification, IEEE Transactions on Cybernetics 44 (10) (2014) $1858-870$.

[45] G. G. H, V. L. C. F, Matrix computations, Vol. 3, JHU Press, 2012.

[46] T. Zhang, Solving large scale linear prediction problems using stochastic gradient descent algorithms, in: Proceedings of the twenty-first international conference on Machine learning, ACM, 2004, p. 116.

[47] I. W. Tsang, J. T. Kwok, P.-M. Cheung, Core vector machines: Fast svm training on very large data sets, J. Mach. Learn. Res. 6 (2005) 363-392.

[48] T. Joachims, Training linear svms in linear time, in: Proceedings of the 12th ACM SIGKDD international conference on Knowledge discovery and data mining, ACM, 2006, pp. 217-226. 
[49] C.-J. Hsieh, K.-W. Chang, C.-J. Lin, S. S. Keerthi, S. Sundararajan, A dual coordinate descent method for large-scale linear svm, in: Proceedings of the 25th international conference on Machine learning, ACM, 2008, pp. 408-415.

[50] A. Bordes, L. Bottou, P. Gallinari, Sgd-qn: Careful quasi-newton stochastic gradient descent, J. Mach. Learn. Res. 10 (2009) 1737-1754.

[51] Z. Zhu, W. Chen, G. Wang, C. Zhu, Z. Chen, P-packsvm: Parallel primal gradient descent kernel svm, in: Data Mining, 2009. ICDM '09. Ninth IEEE International Conference on, 2009, pp. 677-686.

[52] Y.-W. Chang, C.-J. Hsieh, K.-W. Chang, M. Ringgaard, C.-J. Lin, Training and testing low-degree polynomial data mappings via linear svm, J. Mach. Learn. Res. 11 (2010) 1471-1490.

[53] H.-F. Yu, C.-J. Hsieh, K.-W. Chang, C.-J. Lin, Large linear classification when data cannot fit in memory, in: Proceedings of the 16th ACM SIGKDD International Conference on Knowledge Discovery and Data Mining, KDD '10, ACM, New York, NY, USA, 2010, pp. 833-842.

[54] S. Shalev-Shwartz, Y. Singer, N. Srebro, A. Cotter, Pegasos: Primal estimated sub-gradient solver for svm, Mathematical programming 127 (1) (2011) 3-30.

[55] Z. Wang, N. Djuric, K. Crammer, S. Vucetic, Trading representability for scalability: Adaptive multi-hyperplane machine for nonlinear classification, in: Proccedings of the 17th ACM SIGKDD International Conference on Knowledge Discovery and Data Mining(KDD11), 2011, pp. 24-32.

[56] Z. Wang, C. Koby, V. Slobodan, Breaking the curse of kernelization: Budgeted stochastic gradient descent for large-scale SVM training, Journal of Machine Learning Research 13 (1) (2012) 3103-3131.

[57] K. Zhang, L. Lan, Z. Wang, F. Moerchen, Scaling up kernel SVM on limited resources: A low-rank linearization approach., in: Proceedings of the 15 th International Conference on Artificial Intelligence and Statistics (AISTATS), Vol. 22, 2012, pp. 1425-1434. 
[58] C.-J. Hsieh, S. Si, I. S. Dhillon, A divide-and-conquer solver for kernel support vector machines, arXiv preprint arXiv:1311.0914.

[59] A. Rahimi, B. Recht, Weighted sums of random kitchen sinks: Replacing minimization with randomization in learning, in: D. Koller, D. Schuurmans, Y. Bengio, L. Bottou (Eds.), Advances in Neural Information Processing Systems 21, Curran Associates, Inc., 2009, pp. 1313-1320.

[60] Y. Zhai, Y.-S. Ong, I. Tsang, The emerging "big dimensionality", Computational Intelligence Magazine, IEEE 9 (3) (2014) 14-26.

[61] Y. Mu, G. Huay, W. Fan, S.-F. Chang, Hash-SVM: Scalable kernel machines for large-scale visual classification, in: Conference on Visualization and Pattern Recognition(CVPR), 2014, pp. 1-8.

[62] A. Verdaldi, A. Zisserman, Efficient additive kernels via explicit feature maps, IEEE Transactions on Pattern Analysis and Machine Intelligence 34 (3) (2012) 480-492.

[63] S. Paisitkriangkrai, C. Shen, A. van den Hengel, A scalable stagewise approach to large-margin multiclass loss-based boosting, IEEE Transactions on Neural Networks and Learning Systems 25 (5) (2014) 1002-1013.

[64] S. Shalev-Shwartz, T. Zhang, Accelerated proximal stochastic dual coordinate ascent for regularized loss minimization, in: Proceedings of the 31st International Conference on Machine Learning, 2014, pp. 64-72.

[65] A. Krizhevsky, I. Sutskever, G. E. Hinton, Imagenet classification with deep convolutional neural networks, in: Advances in Neural Information Processing Systems(NIPS'2012), 2012, pp. 1-9.

[66] M. Tan, I. W. Tsang, L. Wang, Minimax sparse logistic regression for very highdimensional feature selection, IEEE Transactions on Neural Networks and Learning Systems 24 (10) (2013) 1609-1622. 
[67] Q. Mao, I. W. Tsang, Efficient multi-template learning for structured prediction, IEEE Transactions on Neural Networks and Learning Systems. 24 (2) (2013) 248-261.

[74] E. Carl, Y. Gale, The approximation of one matrix by another of lower rank, Psychometrika 1 (3) (1936) 211-218.

[75] N. Halko, P.-G. Martinsson, J. A. Tropp, Finding structure with randomness: Probabilistic algorithms for constructing approximate matrix decompositions,

[76] D. Achlioptas, F. Mcsherry, Fast computation of low rank matrix approximations, Journal of the ACM 54 (2) (2007) 611-618. 
[77] S. Kumar, M. Mohri, A. Talwalkar, Sampling methods for the Nystrom method, Journal of Machine Learning Research 13 (4) (2012) 981-1006.

[86] K. Choi, K.-A. Toh, H. Byun, Incremental face recognition for large-scale social network services, Pattern Recognition 45 (8) (2012) 2868-2883.

[87] G.-X. Yuan, C.-H. Ho, C.-J. Lin, An improved glmnet for 11-regularized logistic regression, J. Mach. Learn. Res. 13 (1) (2012) 1999-2030. 
[88] D. Chen, W. Chen, Q. Yang, Characterizing inverse time dependency in multiclass learning, in: Proceedings of the 11th IEEE International Conference on Data Mining (ICDM),, 2011, pp. 1020-1025.

[89] F. Chang, C.-Y. Guo, X.-R. Lin, C.-J. Lu, Tree decomposition for large-scale SVM problems, Journal of Machine Learning Research 11 (2010) 2935-2972.

[90] F. Orabona, J. Keshet, B. Caputo, Bounded kernel-based online learning, Journal of Machine Learning Research 10 (2009) 2643-2666.

[91] J. D. M. Rennie, R. Rifkin, Improving multiclass text classification with the support vector machine, Tech. rep. (2001). 